\title{
Stroke Recognition for First Aid Providers: A Systematic Review and Meta-Analysis
}

\author{
Daniel Meyran ${ }^{1,2}$, Pascal Cassan ${ }^{3}$, Bert Avau ${ }^{4}$, Eunice Singletary ${ }^{5}$, David A. Zideman ${ }^{6}$
}

1. Healthcare Division, French Red Cross, Paris, FRA 2. Prehospital Emergency Care, Bataillon De Marins Pompiers De Marseille, Marseille, FRA 3. International Federation of Red Cross and Red Crescent Societies (IFRC) Global First Aid Reference Center, French Red Cross, Paris, FRA 4. Centre for Evidence-Based Practice, Rode Kruis-Vlaanderen, Mechelen, BEL 5. Emergency Medicine, University of Virginia, Charlottesville, USA 6. Pre-Hospital Emergency Medicine, Thames Valley Air Ambulance, Oxford, GBR

Corresponding author: Daniel Meyran, daniel.meyran@numericable.fr

\section{Abstract}

\section{Aim}

To perform a systematic review of the literature on the effectiveness of existing stroke recognition scales used in a prehospital setting and suitable for use by first aid providers. The systematic review will be used to inform an update of international first aid guidelines.

\section{Methods}

We followed the Cochrane Handbook for Systematic Reviews of Interventions methodology and report results according to PRISMA guidelines. We searched Medline, Embase and CENTRAL on May 25, 2020 for studies of stroke recognition scales used by first aid providers, paramedics and nurses for adults with suspected acute stroke in a prehospital setting. Outcomes included change in time to treatment, initial recognition of stroke, survival and discharge with favorable neurologic status, and increased layperson recognition of the signs of stroke. Two investigators reviewed abstracts, extracted and assessed the data for risk of bias. The certainty of evidence was evaluated using GRADE methodology.

\section{Results}

We included 24 observational studies with 10,446 patients evaluating 10 stroke scales (SS). All evidence was of moderate to very low certainty. Use of the Kurashiki Prehospital SS (KPSS), Ontario Prehospital SS (OPSS) and Face Arm Speech Time SS (FAST) was associated with an increased number of suspected stroke patients arriving to a hospital within three hours and, for OPSS, a higher rate of thrombolytic therapy. The KPSS was associated with a decreased time from symptom onset to hospital arrival. Use of FAST Emergency Response (FASTER) was associated with decreased time from door to tomography and from symptom onset to treatment. The Los Angeles Prehospital Stroke Scale (LAPSS) was associated with an increased number of correct initial diagnoses. Meta-analysis found the summary estimate sensitivity of four scales ranged from 0.78 to 0.86 . The FAST and Cincinnati Prehospital Stroke Scale (CPSS) were found to have a summary estimated sensitivity of $0.86,95 \%$ CI [0.69-0.94] and $0.81,95 \%$ CI [0.70-0.89], respectively.

\section{Conclusion}

Stroke recognition scales used in the prehospital first aid setting improves the recognition and diagnosis of stroke, thereby aiding the emergency services to triage stroke victims directly down an appropriate stroke care pathway. Of those prehospital scales evaluated by more than a single study, FAST and Melbourne Ambulance Stroke Screen (MASS) were found to be the most sensitive for stroke recognition, while the CPSS had higher specificity. When blood glucose cannot be measured, the simplicity of FAST and CPSS makes these particular stroke scales appropriate for non-medical first aid providers.

Categories: Emergency Medicine, Neurology

Keywords: stroke, score, prehospital, first aid, triage, recognition scale

\section{Introduction}

Stroke is one of the leading causes of death and disability worldwide [1]. The early detection of stroke in the prehospital setting has the potential to improve stroke outcomes by decreasing delays in treatment. A variety of stroke assessment scales have been developed for both in-hospital and prehospital use. Stroke scales designed for the prehospital setting have a lower number of diagnostic criteria, easy-to-identify clinical signs and simplicity of implementation, making them applicable for use by first aid providers and lay persons. In 2015, the International Liaison Committee on Resuscitation (ILCOR) published a Consensus on Science with Treatment Recommendations (CoSTR), suggesting a benefit from the first aid use of stroke recognition scoring systems or scales for individuals with suspected acute stroke [2, 3].

The objective of this systematic review was to synthesize the evidence for the diagnostic accuracy and clinical effectiveness of stroke scales applied by laypeople, paramedics and nurses in a prehospital setting, according to the research question: Among adults with suspected acute stroke, does the use of a rapid stroke scoring system or scale, compared with basic first aid assessment without the use of a scale, change time to 
treatment, recognition of stroke, discharge with favorable neurologic status, survival with favorable neurologic outcomes, and increase the public/layperson recognition of stroke signs?

\section{Materials And Methods}

This systematic review was conducted in accordance with the Cochrane Handbook for Systematic Reviews of Interventions [4], and reporting occurred through the Preferred Reporting Items for Systematic Reviews and Meta-Analyses (PRISMA) checklist [5]. This review will inform the International Liaison Committee on Resuscitation (ILCOR) consensus on science and treatment recommendations for stroke recognition.

\section{Eligibility criteria and outcomes}

The population included adults over 18 years old, suspected of having a stroke in the prehospital setting, regardless of its type or severity, including ischemic stroke, hemorrhagic stroke or transient ischemic attack (TIA). We excluded all patients with trauma.

The intervention/index test was the use of a single, rapid stroke scale during primary patient assessment to diagnose stroke, as used by a first aid provider, paramedic or nurse. We excluded studies where stroke scales were applied in an emergency department, or assessments made by general practitioners or neurologists. We also excluded stroke scales intended to assess for large vessel occlusion as these were felt to be beyond the skill of a lay first aid provider.

Comparison groups included suspected stroke patients, managed by first aid providers, paramedics or nurses in the prehospital setting who did not use a stroke scale during the primary assessment. To measure the diagnostic accuracy of stroke scales, studies compared the stroke scale result to the hospital diagnosis of stroke as a reference test. An in-hospital diagnosis of stroke was a confirmed documented physician or imaging diagnosis.

The critical outcome was the time to treatment. This outcome included the proportion of patients whose time from symptom onset to hospital arrival or treatment was within two or three hours, time from symptom onset to arrival in the emergency department or hospital, time between hospital arrival to computed tomography (CT) head scan or other imaging ('door' to imaging) and time from symptom onset to administration of tissue Plasminogen Activator (tPA) or the use of endovascular reperfusion techniques.

For the important outcome of recognition of stroke, two types of data studies were eligible: clinical efficacy studies, assessing the proportion of patients receiving appropriate treatment, and diagnostic accuracy studies. Other important outcomes were discharge with favorable neurologic status, survival with favorable neurologic outcome, and cognitive knowledge. The latter outcome evaluated whether stroke recognition scales improve first aid provider recognition of signs of stroke.

\section{Study designs}

Randomized controlled trials (RCTs) and non-randomized studies (non-randomized controlled trials, interrupted time series, controlled before-and-after studies, cohort studies, diagnostic test accuracy studies) were eligible for inclusion. Unpublished studies, conference abstracts, trial protocols and posters were excluded. All languages were included as long as there was an English abstract.

\section{Information sources and search strategy}

We included studies from the 2015 International Liaison Committee on Resuscitation (ILCOR) consensus on first aid science with treatment recommendations (CoSTR) systematic review of stroke assessment scales $[2$, 3]. The existing search strategy, previously run from inception through January 15 , 2015, was re-run in MEDLINE (PUBMED interface), EMBASE (Embase interface), and the Cochrane Central Register of Controlled Trials (CENTRAL) from January 1, 2014 to September 29, 2019 (Appendix A). The search was rerun on May 25, 2020. Additional studies were identified through a hand search of reference lists from included studies.

After removal of duplicates, two authors (PC, DM) independently screened titles and abstracts for relevance. Full texts of potentially relevant publications were retrieved and evaluated by the same reviewers, independently. Papers judged to be relevant were included and reasons for exclusion were documented. Discrepancies between the reviewers were resolved by discussion with the ILCOR First Aid Task Force. Inter-rater reliability was measured with Cohen's kappa at the title and abstract stage and the full text article stage [6].

\section{Data collection}

We used a prespecified data extraction form to collect the following data from included studies: number of participants, age, study characteristics (study design, country, inclusion and exclusion criteria), intervention, training method, reference standard for diagnostic studies, outcome measures and findings. Where possible, missing values were calculated from the available data. For diagnostic studies, we extracted $2 \times 2$ data (true positives, false positives, true negatives and false negatives) directly for each index test.

Risk of bias and certainty of evidence assessment 
For observational studies, the risk of bias (ROB) and certainty of evidence for each individual study was assessed using the Risk Of Bias In Non-randomized Studies of Interventions (ROBINS-I) tool [7]. For diagnostic studies, we assessed the risk of bias of each study using the Quality Assessment of Diagnostic Accuracy Studies version 2 (QUADAS-2) tool [8]. A study was considered at high risk of bias if one of the domains within the ROBINS-I tool or QUADAS-2 tool identified high risk of bias. The Grading of

Recommendations Assessment, Development and Evaluation (GRADE) methodology was used to determine the certainty of evidence for the body of evidence across outcomes [9]. In the GRADE approach, the certainty of evidence can be high, moderate, low or very low. Observational studies assessed with the ROBINS-I tool and diagnostic test accuracy studies assessed with the QUADAS-2 tool start with a high level of certainty [7, 10,11 and can be downgraded across five domains (limitations in study design, imprecision, indirectness, heterogeneity and publication bias), and upgraded across three domains (large magnitude of effect, doseresponse and residual plausible bias and confounding).

\section{Data analysis}

Continuous outcomes are reported as mean differences (MD) with 95\% confidence intervals (CIs). Dichotomous outcomes are reported as risk ratios (RR) with 95\% CIs. There was insufficient data to conduct meta-analyses of effectiveness data. For diagnostic studies, all scales used the same positivity threshold of 'one or greater', which indicates that the person was considered to have a stroke with one or more positive criteria. For each index test, we generated a diagnostic $2 \times 2$ table (true positives, false positives, true negatives and false negatives) from which we calculated sensitivity and specificity with $95 \%$ confidence intervals (CI). When more than one study was identified per scale, we calculated a summary point estimated sensitivity and specificity using a random effects meta-analysis and created Summary Receiver Operating Characteristic (SROC) plots to show the variation in test accuracy estimates across studies with Review Manager 5.3 (RevMan 5.3, The Nordic Cochrane Centre, Copenhagen, Denmark, 2014). Parameter values required by Review Manager Software to construct plots in the SROC space were calculated with MetaDTA: Diagnostic Test Accuracy Meta-Analysis website, version 1.25 (https://crsu.shinyapps.io/dta_ma/) [12].

\section{Results}

For the literature search and study selection, an updated search strategy from 2014 to 2019 and a rerun search strategy from 2019 to 2020 identified 1814 unique titles/abstracts. In addition, we identified new studies and 24 from the previous 2015 search results for the 2015 ILCOR CoSTR for first aid stroke assessment [2]. Based on title and abstract screening, we excluded 1768 studies (reviewer agreement was $95.15 \%$, Kappa $=0.44$ ). Of the 78 full-text articles reviewed, a further 54 were excluded (reviewer agreement was $99.87 \%$, Kappa $=0.79$ ). We ultimately included a total of 24 studies (Figure 1 ). 


\section{Cureus}

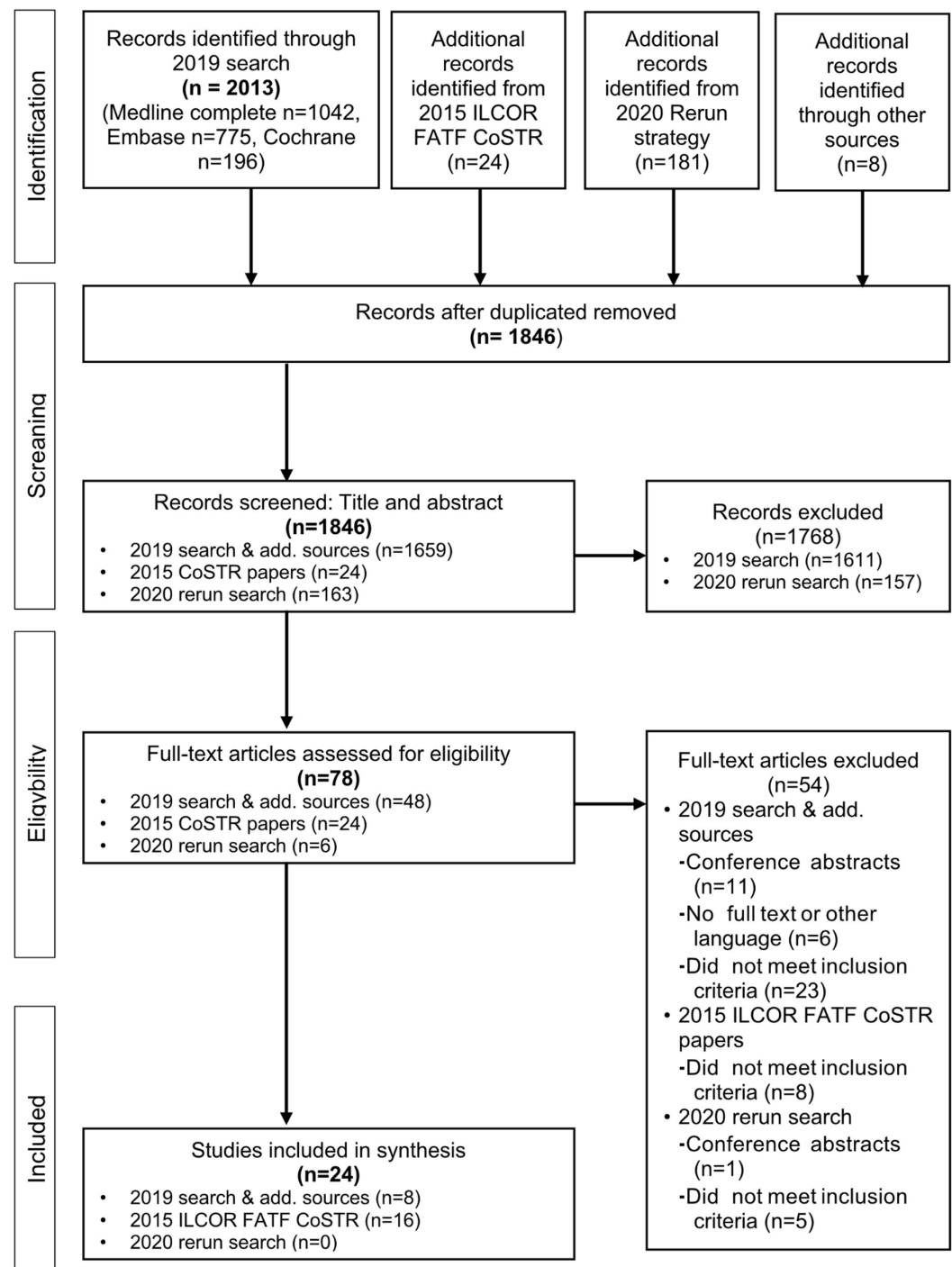

FIGURE 1: PRISMA diagram (diagram illustrating the flow of articles throughout the selection procedures)

CoSTR: Consensus on Science with Treatment Recommendations; FATF: First Aid Task Force; ILCOR: International Liaison Committee on Resuscitation; PRISMA: Preferred reporting items for Systematic Reviews and Meta-Analyses.

\section{Study characteristics}

Characteristics of the included studies are summarized in Table 1. Excluded studies with reasons are presented in Appendix B. We included 24 observational studies; 13 were prospective [13-25], and 11 were retrospectives studies [26-36]. Five studies assessed time to treatment or recognition of stroke outcomes [20, 23, 24, 32, 34], 18 studies assessed the diagnostic accuracy of stroke recognition scales [13-22, 25-28, 30-32, $35,36]$ and one study assessed both time to treatment and diagnostic accuracy [29]. Four studies investigated the "Face, Arm, Speech, Time (FAST)" scale [14, 17, 21, 27], five studies investigated the "Los Angeles Prehospital Stroke Scale (LAPSS)" [14-16, 18, 26], 12 studies investigated the "Cincinnati Prehospital Stroke Scale (CPSS)" [14, 15, 19, 22, 25, 26, 28, 30-32, 35, 36], and three studies investigated the "Melbourne Ambulance Stroke Screen (MASS)" scale [14, 15, 28]. The "Face, Arm, Speech, Time, Emergency Response Protocol (FASTER)” scale, “Ontario Prehospital Stroke Scale (OPSS)”, “Kurashiki Prehospital Stroke Scale (KPSS)", "Recognition of Stroke in the Emergency Room (ROSIER)" scale, "Medic Prehospital Assessment for Code Stroke (MedPACS)", "Balance, eyes, FAST (BeFAST)" and "Prehospital Ambulance Stroke Test (PreHAST)” were investigated by one study each [13, 17, 20, 21, 29, 34, 36]. One study investigated education in stroke signs and symptoms [23]. Sixteen studies investigated only one scale [13, 16, 18-20, 22, 24, 25, 27, 2935] and seven studies investigated two or more scales [14, 15, 17, 21, 26, 28, 36]. The characteristics of stroke recognition scales evaluated in these studies are described in Table 2. 


\begin{tabular}{|c|c|c|c|c|c|c|c|c|}
\hline $\begin{array}{l}\text { (Author, } \\
\text { year) }\end{array}$ & Study design & description & criteria & Scales & standard use & administrator & Training & Outcomes \\
\hline $\begin{array}{l}\text { Andsberg } \\
\text { et al. (2017) } \\
{[13]}\end{array}$ & $\begin{array}{l}\text { Prospective } \\
\text { observational } \\
\text { study }\end{array}$ & $\begin{array}{l}\text { Hässleholm, } \\
\text { Sweden. } \mathrm{N}= \\
69, \text { mean age } \\
\text { not reported. }\end{array}$ & $\begin{array}{l}\text { Inclusion: suspicion } \\
\text { of stroke, defined } \\
\text { as sudden onset of } \\
\text { focal neurologic } \\
\text { symptoms/signs, in } \\
\text { conscious people > } \\
18 \text { years of age. }\end{array}$ & PreHAST & $\begin{array}{l}\text { After reviewing } \\
\text { medical records } \\
\text { by two stroke } \\
\text { physicians. }\end{array}$ & $\begin{array}{l}\text { Ambulance } \\
\text { nurses }\end{array}$ & $\begin{array}{l}\text { Four-hour } \\
\text { education } \\
\text { program } \\
\text { including } \\
\text { practical } \\
\text { training under } \\
\text { supervision and } \\
\text { proper } \\
\text { execution. }\end{array}$ & $\begin{array}{l}\text { Diagnostic } \\
\text { accuracy }\end{array}$ \\
\hline $\begin{array}{l}\text { Asimos et } \\
\text { al. (2014) } \\
{[26]}\end{array}$ & $\begin{array}{l}\text { Retrospective } \\
\text { observational, } \\
\text { cross- } \\
\text { sectional } \\
\text { study }\end{array}$ & $\begin{array}{l}\text { North Carolina, } \\
\text { US. } \mathrm{N}=2442 . \\
\text { Mean age }=66 \\
\text { years (CPSS) } \\
\text { and } 69 \text { years } \\
\text { (LAPSS). } 25.2 \% \\
\text { men. }\end{array}$ & $\begin{array}{l}\text { Inclusion: } \\
\text { preliminary EMS } \\
\text { impression of } \\
\text { stroke. Exclusion: } \\
\text { patients with } \\
\text { duplicate data } \\
\text { records and } \\
\text { patients who were } \\
\text { transferred } \\
\text { between facilities. }\end{array}$ & $\begin{array}{l}\text { CPSS, } \\
\text { LAPSS }\end{array}$ & $\begin{array}{l}\text { ED diagnosis of } \\
\text { stroke, used } \\
\text { ICD } 9 / 10 \text { codes } \\
\text { without any } \\
\text { other detail. }\end{array}$ & Paramedics & Not reported & $\begin{array}{l}\text { Diagnostic } \\
\text { accuracy }\end{array}$ \\
\hline $\begin{array}{l}\text { Bergs et al. } \\
\text { (2010) [14] }\end{array}$ & $\begin{array}{l}\text { Prospective } \\
\text { observational } \\
\text { cross- } \\
\text { sectional } \\
\text { study }\end{array}$ & $\begin{array}{l}\text { Leuven, } \\
\text { Belgium. } \mathrm{N}= \\
135 . \text { Mean age } \\
>77 \text { years. } \\
61 \% \text { men. }\end{array}$ & $\begin{array}{l}\text { Inclusion: all adults } \\
\text { transported with } \\
\text { relevant neurologic } \\
\text { complaints. } \\
\text { Exclusion: ages < } \\
18 \text { years, GCS < } 9 \text {, } \\
\text { transported to } \\
\text { alternate hospital, } \\
\text { trauma, form } \mathrm{t} \\
\text { filled. }\end{array}$ & $\begin{array}{l}\text { FAST, } \\
\text { CPSS, } \\
\text { LAPSS, } \\
\text { MASS }\end{array}$ & $\begin{array}{l}\text { Unspecified, } \\
\text { diagnosis at ED } \\
\text { discharge. }\end{array}$ & $\begin{array}{l}\text { Emergency } \\
\text { nurses }\end{array}$ & $\begin{array}{l}\text { Briefing on } \\
\text { purpose of } \\
\text { study, stroke } \\
\text { scales and } \\
\text { guidelines }\end{array}$ & $\begin{array}{l}\text { Diagnostic } \\
\text { accuracy }\end{array}$ \\
\hline $\begin{array}{l}\text { Berglund et } \\
\text { al. (2014) } \\
\text { [27] }\end{array}$ & $\begin{array}{l}\text { Retrospective } \\
\text { observational } \\
\text { study }\end{array}$ & $\begin{array}{l}\text { Stockholm, } \\
\text { Sweden. } N= \\
900 . \text { Range age } \\
=22-93 \text { years. } \\
55.5 \% \text { men. }\end{array}$ & $\begin{array}{l}\text { Inclusion: all } \\
\text { persons from } 18 \text { to } \\
85 \text { years suspected } \\
\text { of having a stroke } \\
\text { with onset within } \\
\text { six hours and with } \\
\text { independence in } \\
\text { activities of daily } \\
\text { living. }\end{array}$ & FAST & $\begin{array}{l}\text { Diagnosis of } \\
\text { stroke after } \\
\text { imaging, } \\
\text { neurologic } \\
\text { exam, EEG, } \\
\text { laboratory tests. } \\
\text { All participants } \\
\text { received a final } \\
\text { diagnosis by a } \\
\text { neurologist or } \\
\text { stroke } \\
\text { specialist. }\end{array}$ & Paramedics & $\begin{array}{l}\text { One lecture } \\
\text { about stroke } \\
\text { about the FAST } \\
\text { test prior to the } \\
\text { start of the } \\
\text { study. }\end{array}$ & $\begin{array}{l}\text { Diagnostic } \\
\text { accuracy }\end{array}$ \\
\hline $\begin{array}{l}\text { Bray et al. } \\
\text { (2005) [15] }\end{array}$ & $\begin{array}{l}\text { Prospective } \\
\text { observational } \\
\text { cross- } \\
\text { sectional } \\
\text { study }\end{array}$ & $\begin{array}{l}\text { Melbourne, } \\
\text { Australia. } \mathrm{N}= \\
100 .\end{array}$ & $\begin{array}{l}\text { Inclusion: } \\
\text { preliminary EMS } \\
\text { impression of } \\
\text { stroke or suspicion } \\
\text { of stroke by } \\
\text { dispatchers. } \\
\text { Exclusion: not } \\
\text { reported. }\end{array}$ & $\begin{array}{l}\text { CPSS, } \\
\text { LAPSS, } \\
\text { MASS }\end{array}$ & $\begin{array}{l}\text { Diagnosis of } \\
\text { stroke at } \\
\text { discharge } \\
\text { (stroke/TIA } \\
\text { registry) }\end{array}$ & Paramedics & $\begin{array}{l}\text { One-hour } \\
\text { educational } \\
\text { session, and } \\
\text { instruction in } \\
\text { assessment } \\
\text { and } \\
\text { documentation } \\
\text { of items used in } \\
\text { a prehospital } \\
\text { stroke scale. }\end{array}$ & $\begin{array}{l}\text { Diagnostic } \\
\text { accuracy }\end{array}$ \\
\hline \multirow[t]{2}{*}{$\begin{array}{l}\text { Bray et al. } \\
(2010) \text { [28] }\end{array}$} & $\begin{array}{l}\text { Retrospective } \\
\text { observational } \\
\text { Study }\end{array}$ & $\begin{array}{l}\text { Melbourne, } \\
\text { Australia. } \mathrm{N}= \\
850 .\end{array}$ & $\begin{array}{l}\text { Inclusion: patients } \\
\text { with suspicion of } \\
\text { stroke and TIA. } \\
\text { Exclusion: patients } \\
\text { who were } \\
\text { unconscious or } \\
\text { asymptomatic at } \\
\text { the time of } \\
\text { paramedic } \\
\text { assessment. }\end{array}$ & $\begin{array}{l}\text { CPSS, } \\
\text { MASS }\end{array}$ & $\begin{array}{l}\text { Stroke/TIA } \\
\text { registry to } \\
\text { determine if the } \\
\text { discharge } \\
\text { diagnosis was } \\
\text { stroke or TIA. }\end{array}$ & Paramedics & $\begin{array}{l}\text { One-hour } \\
\text { stroke } \\
\text { education } \\
\text { program and } \\
\text { instruction in } \\
\text { the use of } \\
\text { MASS. }\end{array}$ & $\begin{array}{l}\text { Diagnostic } \\
\text { accuracy }\end{array}$ \\
\hline & & $\begin{array}{l}\text { Beijing, China. } \\
N=1130 \text {. Age }\end{array}$ & $\begin{array}{l}\text { Inclusion: patients } \\
\text { suspected of stroke } \\
\text { and TIA. Absence }\end{array}$ & & & & $180 \mathrm{~min}$ training & \\
\hline
\end{tabular}




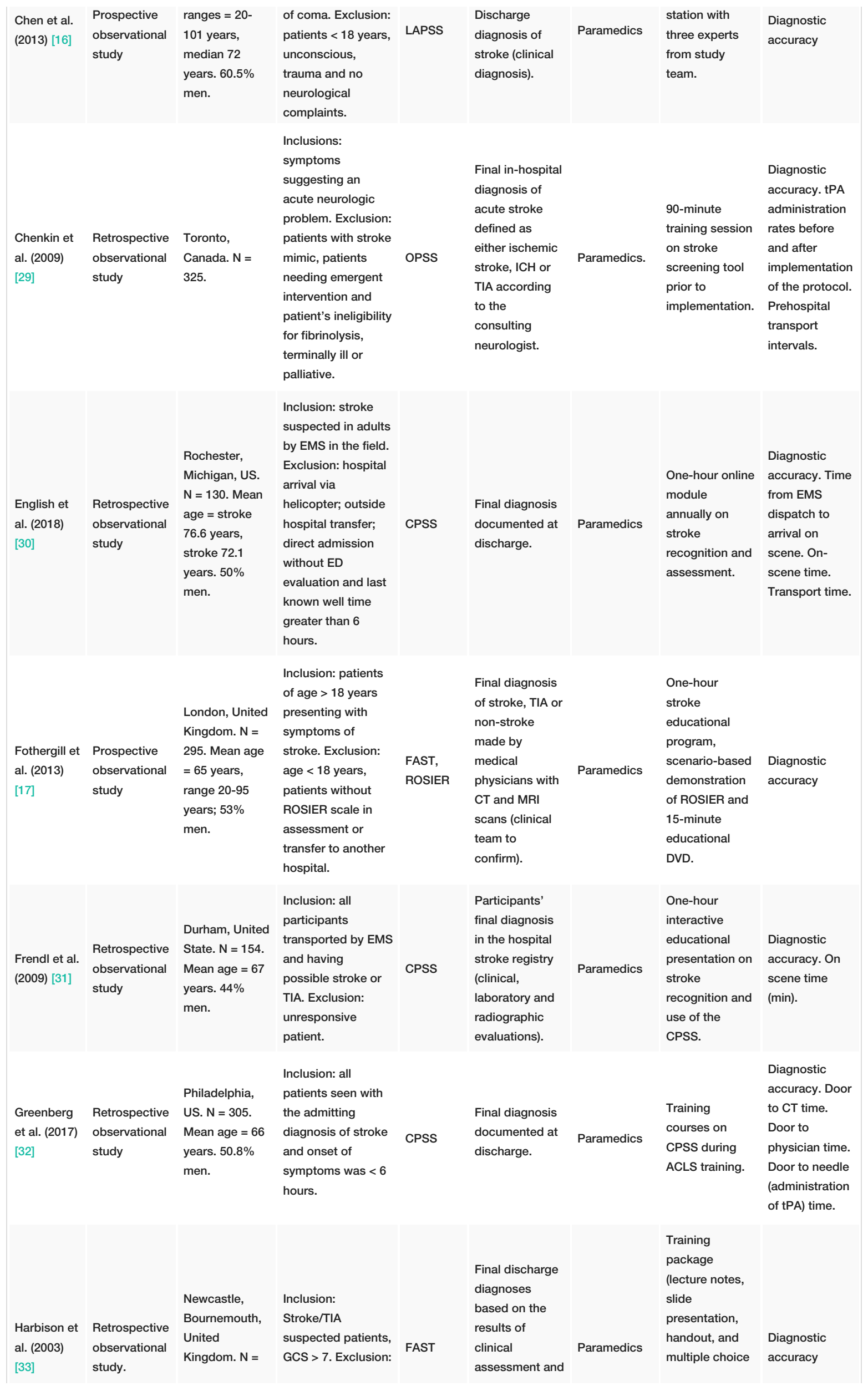




\section{Cureus}

\begin{tabular}{|c|c|c|c|c|c|c|c|c|}
\hline & & $\begin{array}{l}\text { 487. Mean age } \\
=72 \text { years. }\end{array}$ & $\begin{array}{l}\text { subarachnoid } \\
\text { haemorrhage. }\end{array}$ & & $\begin{array}{l}\text { imaging } \\
\text { (Following six } \\
\text { months). }\end{array}$ & & $\begin{array}{l}\text { questionnaire) } \\
\text { presented to } \\
\text { ambulance staff } \\
\text { and newly } \\
\text { recruited staff. }\end{array}$ & \\
\hline $\begin{array}{l}\text { Iguchi et al. } \\
\text { (2010) [34] }\end{array}$ & $\begin{array}{l}\text { Retrospective } \\
\text { observational } \\
\text { study }\end{array}$ & $\begin{array}{l}\text { Kurashiki city, } \\
\text { Japan. } \mathrm{N}=30 . \\
\text { Mean age }=73 \\
\text { years. } 61.9 \% \\
\text { men. }\end{array}$ & $\begin{array}{l}\text { Inclusion: } \\
\text { consecutive } \\
\text { patients transferred } \\
\text { to hospital by } \\
\text { paramedics finally } \\
\text { diagnostic as } \\
\text { having an acute } \\
\text { stroke or TIA within } \\
24 \text { h of onset. }\end{array}$ & KPSS & $\begin{array}{l}\text { Stroke or TIA } \\
\text { was diagnostic } \\
\text { based on the } \\
\text { results of } \\
\text { imaging, MRA } \\
\text { and carotid } \\
\text { duplex } \\
\text { ultrasonography } \\
\text { immediately } \\
\text { after admission. }\end{array}$ & Paramedics & $\begin{array}{l}\text { 90-min training } \\
\text { session }\end{array}$ & $\begin{array}{l}\text { Symptom } \\
\text { onset to } \\
\text { admission time } \\
\text { between } 0 \text { and } \\
3 \text { hours. } \\
\text { Intravenous } \\
\text { tPA. Neurologic } \\
\text { manifestation. } \\
\text { Rate of IV-tPA. } \\
\text { Correlation } \\
\text { between KPSS } \\
\text { (paramedics) } \\
\text { and NIHSS } \\
\text { (neurologist) } \\
\text { after excluding } \\
\text { patients with } \\
\text { onset }>3 \text { hours } \\
\text { before } \\
\text { admission. }\end{array}$ \\
\hline $\begin{array}{l}\text { Kidwell et } \\
\text { al. (2000) } \\
{[18]}\end{array}$ & $\begin{array}{l}\text { Prospective } \\
\text { observational } \\
\text { study }\end{array}$ & $\begin{array}{l}\text { Los Angeles, } \\
\text { US. } \mathrm{N}=206 . \\
\text { Mean age }=63 \\
\text { years. } 52 \% \\
\text { male. }\end{array}$ & $\begin{array}{l}\text { Inclusion: non- } \\
\text { comatose, non- } \\
\text { trauma suspected } \\
\text { strokes in adults } \\
\text { (people with } \\
\text { neurologically } \\
\text { relevant } \\
\text { symptoms). } \\
\text { Exclusion: } \\
\text { asymptomatic upon } \\
\text { EMS arrival, age < } \\
18 \text { years. }\end{array}$ & LAPSS & $\begin{array}{l}\text { Final diagnosis } \\
\text { of stroke at } \\
\text { hospital after a } \\
\text { review of } \\
\text { reports, imaging } \\
\text { and physician } \\
\text { notes. }\end{array}$ & Paramedics & $\begin{array}{l}\text { One-hour initial } \\
\text { training session } \\
\text { with video and } \\
\text { a LAPSS } \\
\text { certification. }\end{array}$ & $\begin{array}{l}\text { Diagnostic } \\
\text { accuracy }\end{array}$ \\
\hline $\begin{array}{l}\text { Kim et al. } \\
\text { (2017) [19] }\end{array}$ & $\begin{array}{l}\text { Prospective } \\
\text { observational } \\
\text { study }\end{array}$ & $\begin{array}{l}\text { Busan, } \\
\text { Republic of } \\
\text { Korea. } \mathrm{N}=268 .\end{array}$ & $\begin{array}{l}\text { Inclusion: patients } \\
\text { with suspected } \\
\text { stroke, patients } \\
\text { who were } \\
\text { transported to } \\
\text { hospital by } \\
\text { paramedics and } \\
\text { patients with true } \\
\text { stroke admitted } \\
\text { during the same } \\
\text { period. }\end{array}$ & CPSS & $\begin{array}{l}\text { Final diagnosis } \\
\text { of stroke or TIA } \\
\text { (no other } \\
\text { mention). }\end{array}$ & Paramedics & Not reported & $\begin{array}{l}\text { Diagnostic } \\
\text { accuracy }\end{array}$ \\
\hline $\begin{array}{l}\text { Kothari et } \\
\text { al. (1999) } \\
{[25]}\end{array}$ & $\begin{array}{l}\text { Prospective } \\
\text { observational } \\
\text { study }\end{array}$ & $\begin{array}{l}\text { Cincinnati, } \\
\text { United states. } \\
\mathrm{N}=171 . \text { Mean } \\
\text { age }=57.8 \\
\text { years. } 72 \% \\
\text { men. }\end{array}$ & $\begin{array}{l}\text { Inclusion: patients } \\
\text { with stroke, TIA, a } \\
\text { stroke-mimicking } \\
\text { condition, or a } \\
\text { combination of } \\
\text { these conditions or } \\
\text { patients with other } \\
\text { neurologic } \\
\text { disorders recruit in } \\
\text { an ED service and } \\
\text { neurology service. }\end{array}$ & CPSS & $\begin{array}{l}\text { CPSS made by } \\
\text { physician. }\end{array}$ & Paramedics & $\begin{array}{l}\text { 10-minute } \\
\text { review on how } \\
\text { to perform } \\
\text { CPSS with } \\
\text { paramedics and } \\
\text { EMTs. Only } \\
\text { verbal } \\
\text { instructions } \\
\text { were given. }\end{array}$ & $\begin{array}{l}\text { Diagnostic } \\
\text { accuracy }\end{array}$ \\
\hline $\begin{array}{l}\text { O'Brien et } \\
\text { al. (2012) } \\
\text { [20] }\end{array}$ & $\begin{array}{l}\text { Prospective } \\
\text { observational } \\
\text { study }\end{array}$ & $\begin{array}{l}\text { Gosford, } \\
\text { Australia. } \mathrm{N}= \\
115 .\end{array}$ & $\begin{array}{l}\text { Inclusion: all } \\
\text { patients with an } \\
\text { initial diagnostic of } \\
\text { acute stroke. }\end{array}$ & FASTER & Not reported. & Paramedics & $\begin{array}{l}\text { Information } \\
\text { about } \\
\text { implementation } \\
\text { FAST protocol. }\end{array}$ & $\begin{array}{l}\text { Proportion of } \\
\text { ischemic } \\
\text { stroke patients } \\
\text { who received } \\
\text { tPA. Symptom } \\
\text { onset to } \\
\text { hospital arrival. } \\
\text { ED door-to-CT } \\
\text { scan. ED door- } \\
\text { to-needle (tPA }\end{array}$ \\
\hline
\end{tabular}




\section{Cureus}

\begin{tabular}{|c|c|c|c|c|c|c|c|c|}
\hline & & & & & & & & $\begin{array}{l}\text { administration). } \\
\text { ED door-to- } \\
\text { Stroke Unit. } \\
\text { Adverse } \\
\text { events. }\end{array}$ \\
\hline $\begin{array}{l}\text { Pickham et } \\
\text { al. (2019) } \\
\text { [21] }\end{array}$ & $\begin{array}{l}\text { Prospective } \\
\text { observational } \\
\text { study }\end{array}$ & $\begin{array}{l}\text { Santa Clara } \\
\text { County } \\
\text { (California), US. } \\
N=359 \text {. }\end{array}$ & $\begin{array}{l}\text { Inclusion: patients } \\
\text { with sudden onset } \\
\text { of neurological } \\
\text { symptoms < } 6 \\
\text { hours from EMS } \\
\text { arrival were } \\
\text { assessed. } \\
\text { Exclusion: patients } \\
\text { presenting directly } \\
\text { to the ED. }\end{array}$ & $\begin{array}{l}\text { FAST, } \\
\text { BEFAST }\end{array}$ & $\begin{array}{l}\text { The patient's } \\
\text { final diagnosis } \\
\text { based on chart } \\
\text { review by } \\
\text { experienced } \\
\text { stroke nurses at } \\
\text { each } \\
\text { participating } \\
\text { hospital. }\end{array}$ & Paramedics & $\begin{array}{l}\text { One-hour } \\
\text { training video. }\end{array}$ & $\begin{array}{l}\text { Diagnostic } \\
\text { accuracy }\end{array}$ \\
\hline $\begin{array}{l}\text { Ramanujam } \\
\text { et al. (2008) } \\
\text { [35] }\end{array}$ & $\begin{array}{l}\text { Retrospective } \\
\text { observational } \\
\text { study }\end{array}$ & $\begin{array}{l}\text { San Diego, } \\
\text { United states. } \\
\mathrm{N}=1045\end{array}$ & $\begin{array}{l}\text { Inclusion: patient } \\
\text { with acute stroke } \\
\text { identification by } \\
\text { EMD or paramedics } \\
\text { and age > } 18 \text { years. } \\
\text { Exclusion: patients } \\
\text { who were taken to } \\
\text { other acute care } \\
\text { hospitals, not } \\
\text { transported by City } \\
\text { EMS agency or with } \\
\text { no final outcome } \\
\text { data. }\end{array}$ & CPSS & $\begin{array}{l}\text { Stroke team } \\
\text { diagnostic or } \\
\text { hospital } \\
\text { discharge } \\
\text { diagnostic. }\end{array}$ & Paramedics & Not reported & $\begin{array}{l}\text { Diagnostic } \\
\text { accuracy }\end{array}$ \\
\hline $\begin{array}{l}\text { Studnek et } \\
\text { al. (2013) } \\
{[36]}\end{array}$ & $\begin{array}{l}\text { Retrospective } \\
\text { observational } \\
\text { study }\end{array}$ & $\begin{array}{l}\text { Charlotte, } \\
\text { North Carolina. } \\
\mathrm{N}=416 . \text { Mean } \\
\text { average age = } \\
66.8 \text { years. } \\
45.7 \% \text { male. }\end{array}$ & $\begin{array}{l}\text { Inclusion: } \\
\text { suspected stroke or } \\
\text { TIA patients who } \\
\text { received a } \\
\text { prehospital } \\
\text { MedPACS screen } \\
\text { and were } \\
\text { transported to one } \\
\text { of the seven local } \\
\text { hospitals. } \\
\text { Exclusion: age < } 18 \\
\text { years, } \\
\text { unconscious, } \\
\text { seizures, no } \\
\text { documented } \\
\text { assessment, } \\
\text { secondary } \\
\text { transports. }\end{array}$ & $\begin{array}{l}\text { CPSS, } \\
\text { MedPACS }\end{array}$ & $\begin{array}{l}\text { Stroke } \\
\text { diagnosis at } \\
\text { hospital } \\
\text { discharge. }\end{array}$ & Nurses & $\begin{array}{l}\text { 2-hour } \\
\text { continuing } \\
\text { education } \\
\text { lecture } \\
\text { regarding } \\
\text { neurologic } \\
\text { emergencies. }\end{array}$ & $\begin{array}{l}\text { Diagnostic } \\
\text { accuracy }\end{array}$ \\
\hline $\begin{array}{l}\text { Vanni et al. } \\
\text { (2011) [22] }\end{array}$ & $\begin{array}{l}\text { Prospective } \\
\text { observational } \\
\text { study }\end{array}$ & $\begin{array}{l}\text { Firenze, Roma, } \\
\text { and Pescara, } \\
\text { Italy. } \mathrm{N}=155 . \\
\text { Mean age = } 72 \\
\text { years. } 59 \% \\
\text { men. }\end{array}$ & $\begin{array}{l}\text { Inclusion: presence } \\
\text { at triage of acute } \\
\text { focal neurological } \\
\text { deficits or a local } \\
\text { EMS dispatch for } \\
\text { suspected stroke. } \\
\text { Exclusion: major } \\
\text { trauma and coma } \\
\text { (GCS < 8). Patients } \\
\text { with terminal } \\
\text { illnesses (life } \\
\text { expectancy < } 3 \\
\text { months). }\end{array}$ & CPSS & $\begin{array}{l}\text { Stroke } \\
\text { diagnoses were } \\
\text { established by a } \\
\text { consensus of } \\
\text { three experts } \\
\text { after reviewing } \\
\text { all clinical data } \\
\text { and imaging } \\
\text { results. }\end{array}$ & Nurses & Not reported. & $\begin{array}{l}\text { Diagnostic } \\
\text { accuracy }\end{array}$ \\
\hline $\begin{array}{l}\text { Wall et al. } \\
\text { (2008) [23] }\end{array}$ & $\begin{array}{l}\text { Prospective } \\
\text { observational } \\
\text { study }\end{array}$ & $\begin{array}{l}\text { Massachusetts, } \\
\text { Boston, United } \\
\text { states. Age = } \\
40 \text { to } 64 \text { years. }\end{array}$ & $\begin{array}{l}\text { Inclusion: Women } \\
\text { from the Well- } \\
\text { Integrated } \\
\text { Screening and } \\
\text { Evaluation for } \\
\text { Women Across the } \\
\text { Nation } \\
\text { (WISEWOMAN). }\end{array}$ & FAST & None & Lay public & $\begin{array}{l}\text { Education } \\
\text { session with 3- } \\
\text { minute } \\
\text { animation to } \\
\text { teach the signs } \\
\text { of stroke. }\end{array}$ & $\begin{array}{l}\text { Knowledge } \\
\text { changes } \\
\text { immediately } \\
\text { after 3-month } \\
\text { training. }\end{array}$ \\
\hline
\end{tabular}




\section{Cureus}

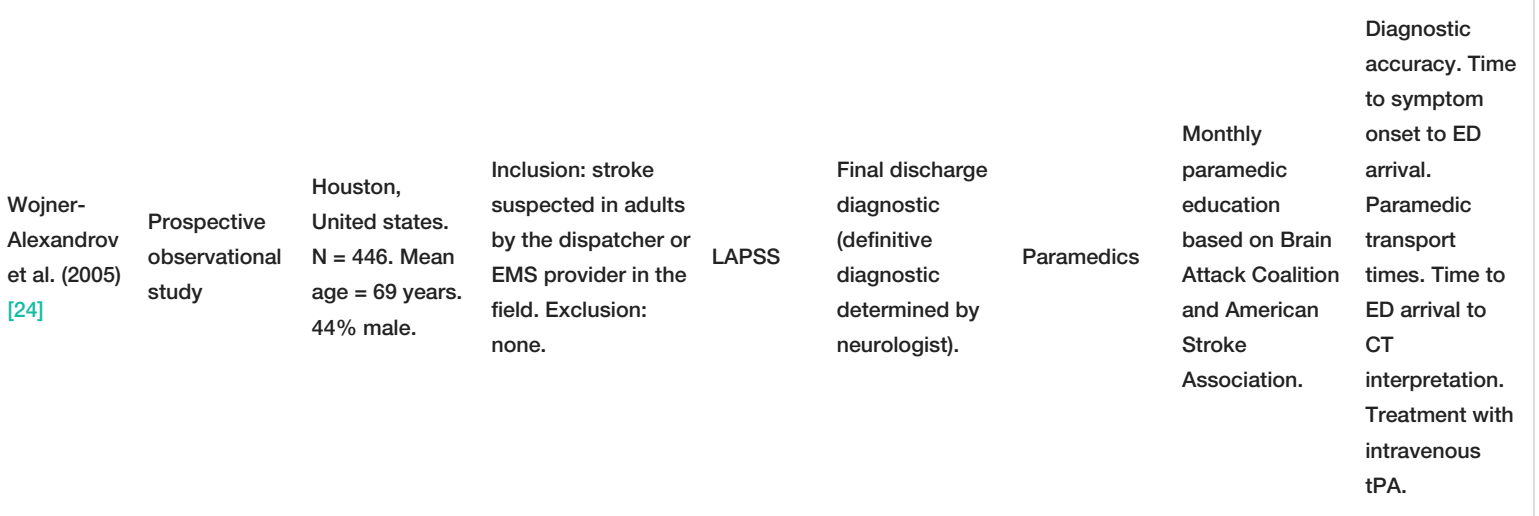

\section{TABLE 1: Characteristics of published meta-analyses}

ACLS: Advanced Cardiac Life Support; CPSS: Cincinnati Prehospital Stroke Scale; CT: Computerised tomography; DVD: Digital Versatile Disc; ED: Emergency Department; EEG: Electroencephalogram; EMD: Emergency Medical Dispatcher; EMS: Emergency Medical Service; EMT: Emergency Medical Technician; FAST: Face Arm Speech Time; FASTER: Face, Arm, Speech, Time, Emergency Response; GCS: Glasgow Coma Scale; ICD: International Classification of Diseases; ICH: Intracerebral Haemorrhage; IV: Intravenous; KPSS: Kurashiki Prehospital Stroke Scale; LAPSS: Los Angeles Prehospital Stroke Scale; MASS: Melbourne Ambulance Stroke Screen; MedPACS: Medic Prehospital Assessment for Code Stroke; MRA: Magnetic Resonance Angiography; MRI: Magnetic Resonance Imaging; NIHSS: National Institute of Health Stroke Score; OPSS: Ontario Prehospital Stroke Scale; PreHAST: PreHospital Ambulance Stroke Test; ROSIER: Recognition of Stroke in the Emergency Room; TIA: Transient Ischemic Attack; tPA: Tissue plasminogen activator. 


\section{Cureus}

\begin{tabular}{|c|c|c|c|c|c|c|c|c|c|c|c|}
\hline Assessment & FAST & CPSS & OPSS & KPSS & ROSIER & MASS & $\begin{array}{l}\text { Med } \\
\text { PACS }\end{array}$ & LAPSS & PreHAST & FASTER & BEFAST \\
\hline $\begin{array}{l}\text { Number of physical examination } \\
\text { items }\end{array}$ & 3 & 3 & 4 & 5 & 5 & 4 & 5 & 3 & 8 & 5 & 5 \\
\hline Facial droop & Yes & Yes & Yes & & Yes & Yes & Yes & Yes & Yes & Yes & Yes \\
\hline Arm weakness/drift & Yes & Yes & Yes & Yes & Yes & Yes & Yes & Yes & Yes & Yes & Yes \\
\hline Leg weakness/drift & & & Yes & Yes & Yes & & Yes & & Yes & & \\
\hline Hand grip strength & & & & & & Yes & & Yes & & & \\
\hline Stability & & & & & & & & & & Yes & \\
\hline Speech difficulty & Yes & Yes & Yes & Yes & Yes & Yes & Yes & & Yes & Yes & Yes \\
\hline Eye position, gaze preference & & & & & & & Yes & & Yes & & \\
\hline Visual field & & & & & Yes & & & & Yes & Yes & \\
\hline Eye diplopia & & & & & & & & & & & Yes \\
\hline Sensory (pain) & & & & & & & & & Yes & & \\
\hline Balance coordination & & & & & & & & & & & Yes \\
\hline Command, verbal instruction & & & & & & & & & Yes $^{1}$ & & \\
\hline Consciousness disturbance & & & & Yes & & & & & & & \\
\hline Level of consciousness & & & & Yes & & & & & & & \\
\hline Score range & $0-3$ & $0-3$ & $0-4$ & $0-13$ & -2 to 5 & $0-4$ & $0-5$ & $0-3$ & $0-19$ & $0-5$ & $0-5$ \\
\hline Eligibility criteria & Yes $^{2}$ & & Yes $^{3}$ & & Yes $^{4}$ & Yes $^{5}$ & Yes $^{6}$ & Yes $^{7}$ & $\mathrm{Yes}^{8}$ & Yes $^{9}$ & Yes \\
\hline Blood glucose measurement & & & Yes & & Yes & Yes & Yes & Yes & & Yes & \\
\hline
\end{tabular}

\section{TABLE 2: Characteristics of prehospital stroke recognition scales}

BEFAST: Balance Eyes Face Arm Speech Time on call; CPSS: Cincinnati Prehospital Stroke Scale; FAST: Face Arm Speech Time; FASTER: Face, Arm, Speech, Time, Emergency Response; KPSS: Kurashiki Prehospital Stroke Scale; LAPSS: Los Angeles Prehospital Stroke Scale; MASS: Melbourne Ambulance Stroke Screen; MedPACS: Medic Prehospital Assessment for Code Stroke; OPSS: Ontario PreHospital Stroke Scale; PreHAST: PreHospital Ambulance Stroke Test; ROSIER: Recognition of Stroke in the Emergency Room.

1. Verbal instruction and sensory, Close your eyes! Grip your hand! (n-paretic side); 2 . GCS $<7$ or suspected head injury exclusion original paper; 3 . Seizure at onset, can be transported to arrive within two hours of onset, time since symptom onset $<2$ hours, GCS $<10$, blood glucose $>4 \mathrm{mmol} / \mathrm{L}$ symptoms of the stroke have resolved; 4 . Blood glucose $>3.5 \mathrm{mmol} / \mathrm{L}$, history of seizure; 5 . History of seizure, time since symptom onset $<24$ hours, at baseline, patient is not wheelchair bound or bedridden, age $>45$ years, blood glucose 2.8 to $22.2 \mathrm{mmol} / \mathrm{L} ; 6$. History of seizure, time since symptom onset < 24 hours, at baseline, patient is not wheelchair bound or bedridden, blood glucose 3.3 to $22.2 \mathrm{mmol} / \mathrm{L} ; 7$. History of seizure, at baseline, patient is not wheelchair bound or bedridden, blood glucose 2.8 to $22.2 \mathrm{mmol} / \mathrm{L}$, age limit $=40$ years; 8 . Age $>18$ years, intended for use, only in conscious people, i.e. alert or aroused by stimulation; 9 . Time of onset less than 2 hours, blood glucose measurement inside the range of $4-$ $17 \mathrm{mmol} / \mathrm{L}$.

\section{Risk of bias within studies and certainty of the evidence}

An overview of the assessment of the overall certainty of evidence, using ROBINS-I assessment tool for nonrandomized studies of interventions studies and QUADAS-2 for diagnostic studies is provided in Tables 3,4 respectively. Overall, the certainty of evidence was moderate to very low across all outcomes, primarily due to risk of bias, indirectness and imprecision. A detailed overview of GRADE assessments per outcome can be found in Appendix C. 


\begin{tabular}{|c|c|c|c|c|c|c|c|c|}
\hline \multirow[b]{2}{*}{ Study (Author, year) } & \multicolumn{8}{|l|}{ Domain } \\
\hline & Confounding & Selection & $\begin{array}{l}\text { Classification of } \\
\text { intervention }\end{array}$ & $\begin{array}{l}\text { Deviation from intended } \\
\text { intervention }\end{array}$ & $\begin{array}{l}\text { Missing } \\
\text { data }\end{array}$ & Outcomes & $\begin{array}{l}\text { Selective } \\
\text { reporting }\end{array}$ & Overall \\
\hline Chenkin et al. (2009) [29] & Serious & Low & Low & Serious & Serious & Low & Low & $\begin{array}{l}\text { Very } \\
\text { serious }\end{array}$ \\
\hline $\begin{array}{l}\text { Harbison et al. (2003) } \\
\text { [33] }\end{array}$ & Information & Low & Serious & Low & Low & Low & Low & $\begin{array}{l}\text { Very } \\
\text { serious }\end{array}$ \\
\hline Iguchi et al. (2011) [34] & Low & Serious & Low & Low & Serious & Moderate & Low & $\begin{array}{l}\text { Very } \\
\text { serious }\end{array}$ \\
\hline $\begin{array}{l}\text { Wojner-Alexandrov et al. } \\
\text { (2005) [24] }\end{array}$ & Low & Serious & Low & Low & Low & Low & Low & Serious \\
\hline O'Brien et al. (2012) [20] & Serious & Serious & Low & Low & Low & Serious & Low & $\begin{array}{l}\text { Very } \\
\text { serious }\end{array}$ \\
\hline Wall et al. (2008) [23] & Low & Low & Low & Low & Low & Low & Low & Low \\
\hline
\end{tabular}

TABLE 3: Risk of bias in non-randomized studies of interventions (ROBINS-I)

\begin{tabular}{|c|c|c|c|c|c|c|c|}
\hline \multirow[b]{2}{*}{ Study (Author, year) } & \multicolumn{4}{|c|}{ Risk of bias } & \multicolumn{3}{|c|}{ Applicability concerns } \\
\hline & $\begin{array}{l}\text { Patient } \\
\text { selection }\end{array}$ & $\begin{array}{l}\text { Index } \\
\text { test }\end{array}$ & $\begin{array}{l}\text { Reference } \\
\text { standard }\end{array}$ & $\begin{array}{l}\text { Flow and } \\
\text { timing }\end{array}$ & $\begin{array}{l}\text { Patient } \\
\text { selection }\end{array}$ & $\begin{array}{l}\text { Index } \\
\text { test }\end{array}$ & $\begin{array}{l}\text { Reference } \\
\text { standard }\end{array}$ \\
\hline Andsberg et al. (2017) [13] & Low & Low & Low & Low & Low & Low & Low \\
\hline Asimos et al. (2014) [26] & High & Low & High & Low & Low & Low & Low \\
\hline Bergs et al. (2010) [14] & High & Low & Unclear & Unclear & Low & Low & Low \\
\hline Bray et al. (2005) [15] & High & Low & Unclear & Unclear & Low & Low & Low \\
\hline Berglund et al. (2014) [27] & Low & Low & Low & Low & Low & Low & Low \\
\hline Bray et al. (2010) [28] & High & Low & Unclear & Unclear & Low & Low & Low \\
\hline Chen et al. (2013) [16] & High & Low & Low & Unclear & Low & Low & Low \\
\hline Chenkin et al. (2009) [29] & High & Low & Unclear & Unclear & Low & Low & Low \\
\hline English et al. (2018) [30] & High & Low & Unclear & Unclear & Low & Low & Low \\
\hline Fothergill et al. (2013) [17] & High & Low & Unclear & Low & Low & Low & Low \\
\hline Frendl et al. (2009) [31] & High & Low & Unclear & Unclear & Low & Low & Low \\
\hline $\begin{array}{l}\text { Greenberg et al. (2016) } \\
\text { [32] }\end{array}$ & Low & Low & Low & High & Low & Low & Low \\
\hline Kothari et al. (1999) [25] & Unclear & Low & Low & Low & Low & Low & Low \\
\hline Kidwell et al. (2000) [18] & Low & Low & Low & Unclear & Low & Low & Low \\
\hline Kim et al. (2017) [19] & High & Low & Unclear & Unclear & Low & Low & Low \\
\hline Pickham et al. (2019) [21] & High & Low & High & Low & Low & Low & Low \\
\hline $\begin{array}{l}\text { Ramanujam et al. (2008) } \\
\text { [35] }\end{array}$ & High & Low & Unclear & Unclear & Low & Low & Low \\
\hline Studnek et al. (2013) [36] & High & Low & Unclear & Unclear & Low & Low & Low \\
\hline Vanni et al. (2011) [22] & Low & Low & Low & Low & Low & Low & High \\
\hline
\end{tabular}

Study findings on stroke assessment scale effectiveness 
For the critical outcome "time to treatment", we identified four observational studies [20, 24, 29, 34] evaluating four different stroke scales (KPSS, LAPSS, OPSS, FASTER). For the KPSS, one retrospective observational study [34], enrolling 430 participants with suspected acute stroke in the prehospital setting, showed an association between the use of KPSS and an increase in the number of patients whose time from symptom onset to hospital arrival was within 3 hours. Of patients who had the KPSS applied, 161/256 (62.9\%) arrived within 3 hours compared with 91/174 (52.3\%) who did not have the scale applied (RR 1.2; 95\% CI [1.01 - 1.43]; $\mathrm{p}=0.034$ : very low certainty evidence). The same study showed significantly shorter elapsed time from symptom onset to hospital admission with the use of KPSS (mean time 2.1 hours; interquartile range [1.0 - 6.2]), compared with no KPSS use (mean time 2.7 hours; interquartile range [1.2 - 9.7]; $p=0.024$; very low certainty evidence). For the LAPSS, one observational study [24], enrolling 1518 prehospital participants with suspected acute stroke, showed an association between the use of the LAPSS and an increased time from symptom onset to emergency department arrival (MD $132.00 \mathrm{~min}$; 95\% CI [14.68 249.32]; $\mathrm{p}=0.097$; very low certainty evidence). The same study did not find a significant benefit associated between use of LAPSS and the proportion of patients admitted within $120 \mathrm{~min}$ (RR 1.07; 95\% CI [0.96 - 1.19]; $\mathrm{p}=0.215$; very low certainty evidence). For OPSS, one observational study [29], enrolling 861 prehospital participants with acute suspected stroke, showed an association between use of the OPSS and increased proportion of patients with a time from symptom onset to hospital arrival within 3 hours when using the OPSS, compared with not using the OPSS (RR 1.43; 95\% CI [1.12 - 1.82]; $\mathrm{p}=0.004$; very low certainty evidence). For FASTER, one observational study [20], enrolling 115 prehospital participants, showed an association between use of FASTER and a shortened time from symptom onset to treatment with tissue Plasminogen Activator (tPA) (MD -32 min; 95\% CI [-53 to -11]; $\mathrm{p}=0.005$; very low certainty of evidence). Furthermore, this study showed an association between the use of FASTER and a shorter door to CT time for patients receiving tPA (MD - $30 \mathrm{~min}$; 95\% CI [-49 to -11] p = 0.004, very low certainty of evidence), and a shorter "door to needle" time for patients receiving tPA (MD -46 min; 95\% CI [-71 to -21] p $=0.001$, very low certainty of evidence). Among patients receiving tPA, no significant differences were found between the groups with or without FASTER applied for time from symptom onset to hospital arrival (MD, $17 \mathrm{~min}$; $95 \% \mathrm{CI}$ [-7 to 41$] ; p=0.180$, very low certainty of evidence). We did not identify any comparative studies evaluating the other scales (FAST, ROSIER, MASS, CPSS, MedPACS and PreHAST) for the critical outcome "time to treatment".

For the important outcome "recognition of stroke" (outcome defined as definitive stroke diagnosis or therapy administration), we identified five observational studies [20, 24, 29, 33, 34] evaluating five different stroke scales (FAST, KPSS, FASTER, OPSS, LAPSS). For the FAST scale, one observational study [33], enrolling 356 prehospital participants with suspected acute stroke, showed an association with use of FAST and an increased proportion of patients with confirmed stroke or TIA admitted within 3 hours following symptom onset (RR 3.3; 95\% CI [2.29 - 4.75]; p < 0.00001, low certainty evidence). For KPSS, one observational study [34], enrolling 430 prehospital participants with suspected acute stroke, showed no difference between use and non-use of KPSS for the proportion of patients who were diagnosed with stroke and received thrombolytic therapy (RR 0.95; 95\% CI [0.59 - 1.53]; $\mathrm{p}=0.838$, low certainty evidence). For LAPSS, one observational study [24], enrolling 1518 prehospital participants, showed an association with the use of LAPSS by paramedics and an increased proportion of correct initial diagnoses of stroke as confirmed by a neurologist (RR 1.29; 95\% CI [1.18 - 1.42]; p < 0.00001, moderate certainty evidence). However, no association was found with the use of the LAPSS and the proportion of patients treated with intravenous tPA among confirmed stroke cases (RR 1.13; 95\% CI [0.71 - 1.80]; $\mathrm{p}=0.601$, moderate certainty evidence). For OPSS, one observational study [29], enrolling 861 prehospital participants, showed no association between the use of OPSS and the rate of recognition of ischemic stroke (RR 1.11; 95\% CI [0.96 - 1.28]; $\mathrm{p}=$ 0.157 , low certainty evidence), but did show an association between the use of OPSS and an increased rate of thrombolytic therapy in ischemic stroke cases (RR 1.72; 95\% CI [1.03 - 2.88]; p = 0.037, low certainty evidence). For FASTER, one observational study [20], including 182 participants, showed an association between the use of FASTER and an increased proportion of stroke patients who received thrombolytic therapy (RR 2.56; 95\% CI [1.02 - 6.45]; $\mathrm{p}=0.045$, very low certainty evidence).

For the important outcome of increased public/layperson recognition of stroke signs, one observational study [23], enrolling 72 participants (members of the public), was included. This study reported that immediately after training compared with pre-training, there was a significant increase in the percentage of participants who recognized facial droop, arm weakness and slurred speech as signs of stroke (68/72 (94.4\%) compared with 55/72 (76.4\%); RR 1.24; 95\% CI [1.07 - 1.42]; p = 0.003, moderate certainty evidence). Of the 65 participants who were retested three months after the training, compared with pre-training, $100 \%$ remembered slurred speech and facial drooping as stroke symptoms; $98.5 \%$ remembered arm weakness or numbness, showing no significant change from the immediate post-training test (moderate certainty of evidence).

We did not identify any comparative studies evaluating stroke recognition for the outcomes of "favorable neurologic status" or "survival with favorable neurologic outcome".

For the outcome of recognition of stroke (diagnostic studies, outcome defined as correct stroke diagnosis), we identified 19 observational studies [13-19, 21, 22, 25-32, 35, 36] including a total of 8153 participants, evaluating nine different screening tools (FAST, LAPSS, OPSS, CPSS, ROSIER, MASS, BEFAST, Med-PACS, Pre-HAST) (Table 5). The reported prevalence, sensitivity, specificity, positive and negative likelihood ratio for each scale are reported in Table 5. Four scales, FAST (Figure 2A), LAPSS (Figure 2B), CPSS (Figure 2C) and MASS (Figure 2D), were assessed by more than one study. The diagnostic accuracy of the FAST scale was assessed by very low certainty evidence from four observational prospective studies [14, 17, 21, 27], including 1585 participants suspected of having a stroke. The summary estimate for sensitivity was $0.86,95 \%$ CI [0.69$0.94]$ and the summary estimate for specificity was $0.38,95 \%$ CI $[0.16-0.66]$. The diagnostic accuracy of the 
LAPSS was assessed by low certainty evidence from four prospective observational studies [14-16, 18] and one retrospective study [26]. The studies included a total of 2692 participants suspected of having a stroke. The summary estimate for sensitivity was $0.78,95 \%$ CI [0.75-0.81] and the summary estimated diagnostic specificity was $0.86,95 \% \mathrm{CI}$ [0.67-0.95]. The diagnostic accuracy of the CPSS was assessed by very low certainty evidence from six prospective observational studies $[14,15,19,22,25,28]$ and six retrospective observational studies [26, 30-32, 35, 36]. The studies included a total of 4842 participants suspected of having a stroke. The summary estimate for sensitivity was $0.81,95 \%$ CI [0.70-0.89] and the summary estimate for specificity was $0.55,95 \%$ CI [0.39-0.69]. Two additional studies were identified [22, 32], but these provided incomplete data and could not be included in the meta-analysis. The diagnostic accuracy of the MASS was assessed by low certainty evidence from two prospective observational studies $[14,15]$ and one retrospective observational study [28]. These three studies included a total of 981 participants suspected of having a stroke. The summary estimate for sensitivity was $0.85,95 \%$ CI $[0.79-0.90]$ and the summary estimate for specificity was 0.82 , 95\% CI [0.69-0.91].

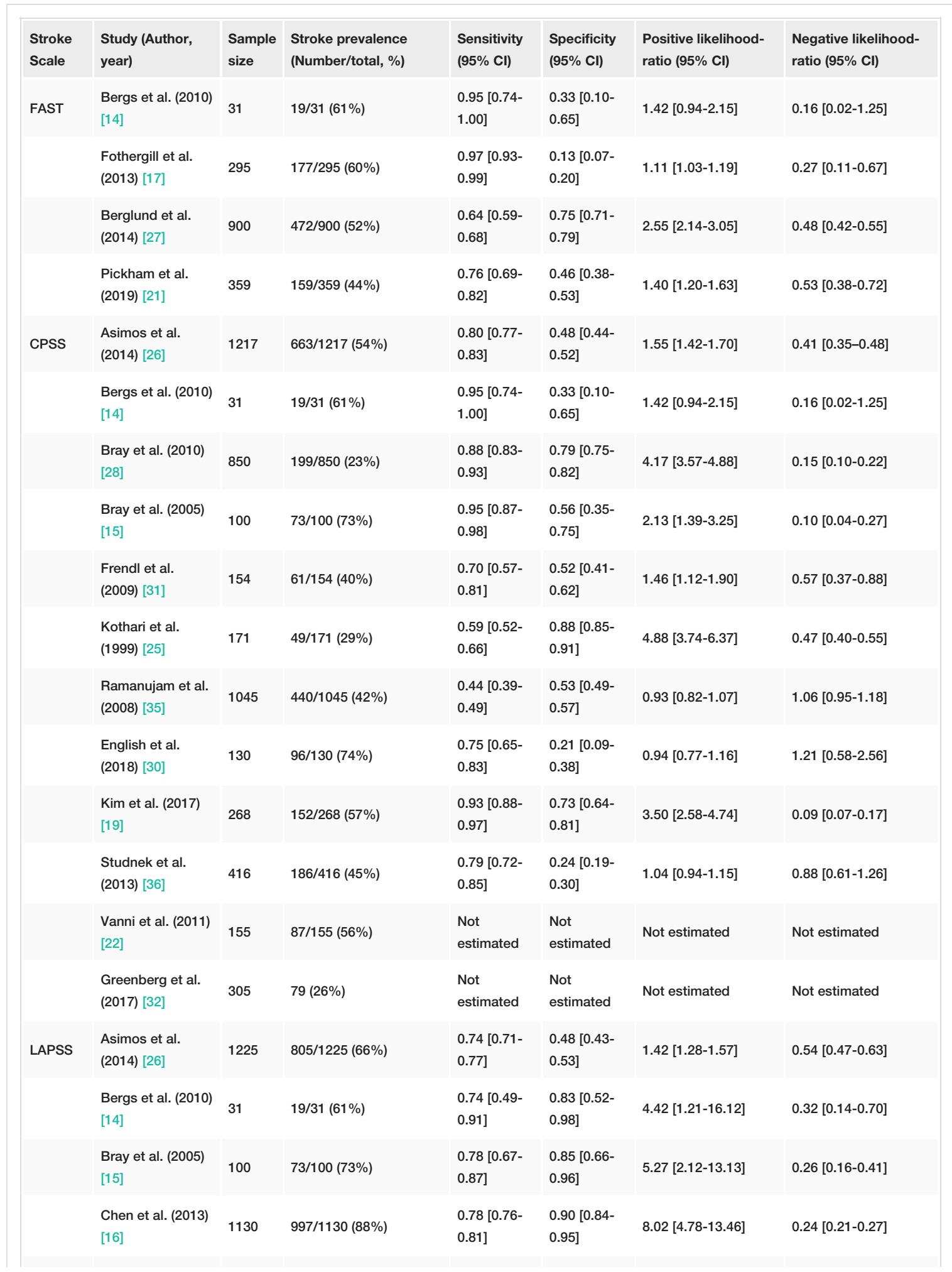




\section{Cureus}

\begin{tabular}{|c|c|c|c|c|c|c|c|}
\hline & $\begin{array}{l}\text { Kidwell et al. } \\
(2000) \text { [18] }\end{array}$ & 206 & $34 / 206$ (16\%) & $\begin{array}{l}0.91[0.76- \\
0.98]\end{array}$ & $\begin{array}{l}0.97[0.93- \\
0.99]\end{array}$ & 31.36 [13.14-74.87] & $0.09[0.03-0.27]$ \\
\hline \multirow[t]{3}{*}{ MASS } & $\begin{array}{l}\text { Bergs et al. (2010) } \\
\text { [14] }\end{array}$ & 31 & 19/31 (61\%) & $\begin{array}{l}0.74[0.49- \\
0.91]\end{array}$ & $\begin{array}{l}0.67[0.35- \\
0.90]\end{array}$ & $2.21[0.95-5.14]$ & $0.39[0.17-0.93]$ \\
\hline & $\begin{array}{l}\text { Bray et al. (2010) } \\
{[28]}\end{array}$ & 850 & 199/850 (23.4\%) & $\begin{array}{l}0.83[0.78- \\
0.88]\end{array}$ & $\begin{array}{l}0.86[0.83- \\
0.88]\end{array}$ & $5.90[4.84-7.20]$ & $0.19[0.14-0.26]$ \\
\hline & $\begin{array}{l}\text { Bray et al. (2005) } \\
{[15]}\end{array}$ & 100 & 73/100 (73\%) & $\begin{array}{l}0.90[0.81- \\
0.96]\end{array}$ & $\begin{array}{l}0.74[0.54- \\
0.89]\end{array}$ & 3.49 [1.84-6.63] & $0.13[0.06-0.27]$ \\
\hline $\begin{array}{l}\text { Med } \\
\text { PACS }\end{array}$ & $\begin{array}{l}\text { Studnek et al. } \\
\text { (2013) [36] }\end{array}$ & 416 & $186 / 416(45 \%)$ & $\begin{array}{l}0.74[0.67- \\
0.80]\end{array}$ & $\begin{array}{l}0.33[0.27- \\
0.39]\end{array}$ & $1.10[0.97-1.25]$ & $0.79[0.58-1.08]$ \\
\hline OPSS & $\begin{array}{l}\text { Chenkin et al. } \\
\text { (2009) [29] }\end{array}$ & 554 & 214/554 (39\%) & $\begin{array}{l}0.87[0.82- \\
0.92]\end{array}$ & $\begin{array}{l}0.59[0.54- \\
0.65]\end{array}$ & $2.15[1.87-2.47]$ & $0.21[0.15-0.31]$ \\
\hline ROSIER & $\begin{array}{l}\text { Fothergill et al. } \\
\text { (2013) [17] }\end{array}$ & 295 & $177 / 295(60 \%)$ & $\begin{array}{l}0.97[0.93- \\
0.99]\end{array}$ & $\begin{array}{l}0.18[0.11- \\
0.26]\end{array}$ & $1.18[1.08-1.28]$ & $0.19[0.08-0.46]$ \\
\hline PreHAST & $\begin{array}{l}\text { Andsberg et al. } \\
(2017) \text { [13] }\end{array}$ & 69 & 26/69 (38\%) & $\begin{array}{l}1.00[0.87- \\
1.00]\end{array}$ & $\begin{array}{l}0.40[0.25- \\
0.56]\end{array}$ & $1.65[1.30-2.11]$ & 0.00 \\
\hline BEFAST & $\begin{array}{l}\text { Pickham et al. } \\
\text { (2019) [21] }\end{array}$ & 359 & $159 / 359(44 \%)$ & $\begin{array}{l}0.91[0.86- \\
0.95]\end{array}$ & $\begin{array}{l}0.26[0.20- \\
0.33]\end{array}$ & $1.23[1.12-1.36]$ & $0.34[0.19-0.59]$ \\
\hline
\end{tabular}

\section{TABLE 5: Operating characteristics of prehospital stroke scales by included study}

FAST: Face Arm Speech Time; CPSS: Cincinnati Prehospital Stroke Scale; LAPSS: Los Angeles Prehospital Stroke Scale; MASS: Melbourne Ambulance Stroke Screen; Med PACS: Medic Prehospital Assessment for Code Stroke; OPSS: Ontario PreHospital Stroke Scale; ROSIER: Recognition of Stroke in the Emergency Room; PreHAST: PreHospital Ambulance Stroke Test; BEFAST: Balance Eyes Face Arm Speech Time on call. 


\section{Cureus}
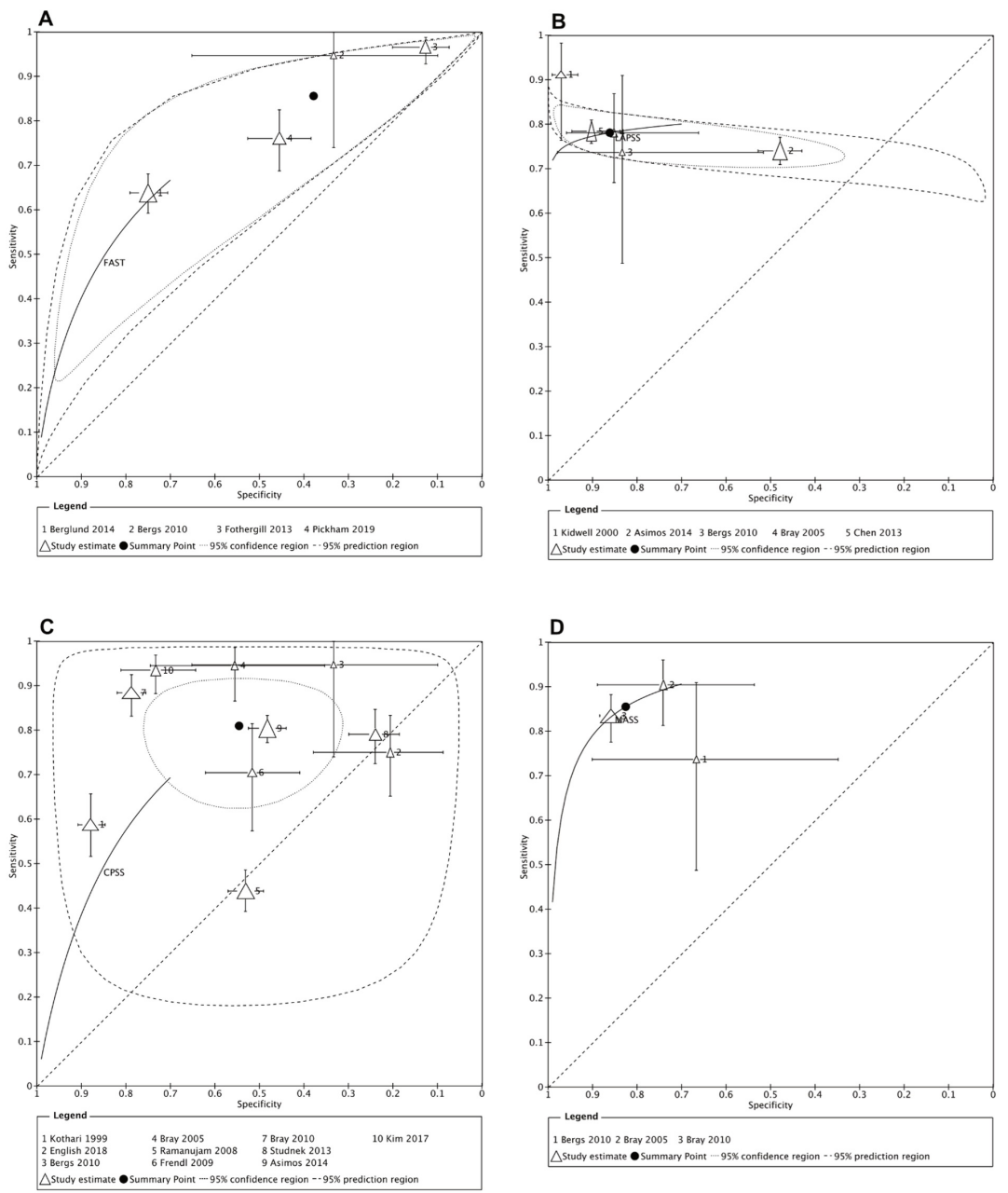

\section{FIGURE 2: Summary receiver operating characteristics plots}

A- Face Arm Speech Time (FAST); B- Los Angeles Prehospital Stroke Scale (LAPSS); C- Cincinnati Prehospital Stroke Scale (CPSS); D- Melbourne Ambulance Stroke Scale (MASS).

Studies of stroke assessment scales can be divided into subgroups based on whether the scale includes blood glucose measurement or not. In the nine diagnostic studies that used stroke scales with blood glucose measurement (LAPSS, OPSS, ROSIER, MASS, Med-PACS) [14-18, 26, 28, 29, 36], the reported sensitivities ranged from 0.74 to 0.97 , compared with 0.80 to 1.00 in the 14 studies of stroke scales that did not include blood glucose measurement (FAST, CPSS, Pre-HAST, BEFAST) [13-15, 17, 19, 21, 25-28, 30, 31, 35, 36]. The reported specificities from studies with stroke scales including blood glucose measurement (LAPSS, OPSS, ROSIER, MASS, Med-PACS) ranged between 0.18 and 0.86 compared with 0.26 to 0.55 in the studies that used scales without blood glucose measurement (PreHAST, FAST, CPSS, BEFAST). The comparison of Summary Receiver Operating Characteristics (SROC) curve between stroke scales with blood glucose measurement and stroke scales without blood glucose measurement is presented in Figure 3. The first comparison covers all studies (Figure $3 A, 3 B$ ); the second covers only the scores assessed by more than one study (Figure 3C, 3D). 


\section{Cureus}
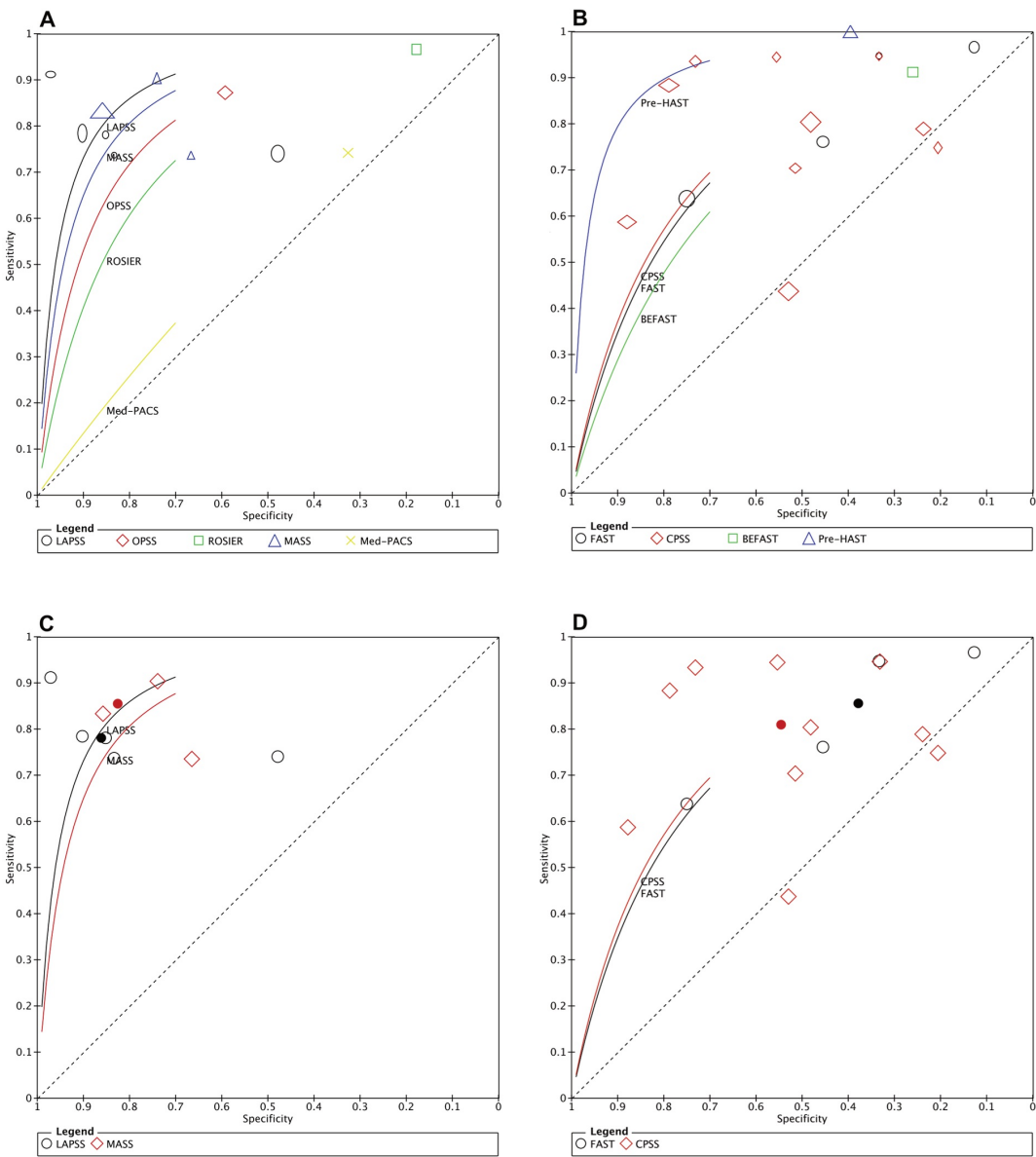

\section{FIGURE 3: Summary receiver operating characteristics (SROC) plot of strokes scales with and without glucose measurement}

A- SROC of stroke scales with glucose measurement; B- SROC of stroke scales without glucose measurement; C- SROC of stroke scales with more than one study per scale with glucose measurement; D- SROC of stroke scales with more than one study per scale without glucose measurement.

\section{Discussion}

We identified and systematically reviewed studies of accuracy for prehospital stroke recognition tools that are applied in the prehospital setting and potentially suitable for use by first aid providers. We consider an ideal stroke assessment tool for first aid to be one that is easily understood and remembered, has a high sensitivity for detecting stroke and can be completed in minimal time. Because the home use of blood glucose measurement devices is increasingly common in populations at risk for acute stroke, we included prehospital stroke scales that incorporate blood glucose measurements but evaluated them separately for accuracy. In this systematic review, three of the four included scales (KPSS, FASTER and OPSS) showed an association between prehospital use and a decreased time from stroke onset to treatment [20, 29, 34]. Unfortunately, it was not possible to perform a meta-analysis for this outcome due to the limited number of studies.

In terms of definitive stroke diagnosis or therapy administration, using a stroke recognition scale in the prehospital setting does not seem to increase the proportion of patients with confirmed stroke diagnosis. However, patients with confirmed stroke were promptly admitted to a hospital and received treatment more quickly.

For accuracy of recognition of stroke we pooled the data from the 17 diagnostic studies of FAST, CPSS, LAPSS, and MASS individually to calculate a summary estimated sensitivity and specificity $[14-19,21,22$, $25-28,30-32,35,36]$. Other scales that were only assessed by a single study were not included $[13,17,21,29$ 36]. We considered both the FAST and CPSS to be stroke assessment tools that a first aid provider would find easy to understand, remember and to use. These two stroke scales are supported by multiple studies with a large total number of participants but do not include a blood glucose measurement. For FAST, the sensitivities in four studies ranged from 0.64 to $0.97[14,17,21,27]$ with a summary estimated sensitivity of 0.86 [0.69-0.94]. For CPSS, the sensitivity measurements from 10 included studies ranged from 0.44 to 
$0.97[14,15,19,25,26,28,30,31,35,36]$ with a summary estimated sensitivity of 0.78 [0.75-0.81]. Specificities of these two scales were significantly less, ranging from 0.13 to 0.88 , with a lower summary estimated specificity for FAST compared with CPSS (0.38 [0.16-0.66] compared with 0.86 [0.67-0.95]). Thus, whilst FAST appears to be a more sensitive scale for the recognition of stroke, it is less specific than the CPSS. The high risk of bias and between-study heterogeneity did not allow us to determine summary estimates sensitivity and specificity of scales with and without blood glucose measurement. Some studies reported a high specificity of above 0.90 , where the proportion of missed stroke patients assessed by the scale ranged from 10 to $22 \%[16,18]$. Prehospital stroke recognition scales should not be interpreted as confirmatory diagnostic tests but only as a screening test. Most of the studies only assessed the outcomes of true positive patients, however it would be of value to know the impact of the scale on those who were false negatives.

Two stroke assessment scales that include blood glucose measurement in their eligibility criteria (MASS and LAPSS) were evaluated by multiple studies and included 981 patients for MASS and 2692 patients for LAPSS (Table 5). We found these scales had similar sensitivities for stroke identification as for scales without blood glucose measurement, but increased specificities (Figure 3C, 3D). We recognize that many first aid providers may not have access or the skills to use a properly calibrated glucometer. Local guidelines would need to determine the benefit of increased specificity of stroke scales that include glucose measurement compared with using simpler stroke scales that do not require glucose measurement.

Three systematic reviews analyzed stroke recognition instruments in the prehospital setting [37-39]. Brandler et al. in 2014 included studies in which the scales were used by paramedics or emergency medical technicians (EMTs) and included scales requiring blood glucose measurement [37]. The authors concluded that LAPSS performed more consistently and that LAPSS and CPSS had similar diagnostic capabilities. Our systematic review includes all of the studies evaluated by Brandler et al. and adds new data from 16 more publications. Additionally, we report diagnostic accuracy of scales that require blood glucose measurement separately from those without glucose measurement, to help identify appropriate scales for use by first aid providers. A systematic review by Rudd et al. in 2016 included all studies in which the scales were administered face-to-face by any prehospital or hospital clinician to identify adults suspected of stroke [38]. Eleven studies included in this systematic review were also included in our review, but 10 studies did not meet our inclusion criteria (seven papers and three abstracts). The authors concluded that available data do not allow a strong recommendation to be made about the superiority of a particular stroke recognition scale evaluated. Zhelev et al. in a Cochrane review in 2019 analyzed prehospital stroke scales as screening tools for early identification of stroke and transient ischemic attack [39]. They included in a "prehospital setting subgroup" all studies where the scale had been used in the prehospital setting regardless of the background and training of the person performing the assessment, and only evaluated diagnostic accuracy. The author concluded, "in the field, CPSS had consistently the highest sensitivity but was less specific than most of the scales". In our systematic review, we have focused on the scales that can potentially be used by trained first aid providers or lay persons in a prehospital setting. We attribute our inclusion and exclusion criteria to any differences in our results. Lastly, our systematic review is not limited to a diagnostic accuracy review. We also evaluated the influence of stroke scale use on the time to treatment and the rate of stroke diagnosis.

Our review has some limitations. First, only four stroke scales were investigated by more than a single study, for which a large number of participants have been included (FAST, CPSS, LAPSS, MASS). Six scales (FASTER, OPSS, KPSS, ROSIER, BEFAST, Med-PACS) were only investigated in single studies, including between 250 and 600 participants $[13,17,20,21,29,34,36]$. The PreHAST scale provided the highest sensitivity $(1.00$, $95 \%$ CI [0.87-1.00]), but was only evaluated in a single study, with 69 participants [13]. The prevalence of stroke/TIA ranged from 23\% [28] to $88 \%$ [16] (Table 5), reflecting differences in population and patient selection that may affect sensitivity and specificity estimates. Second, the accuracy of the scales for identifying people with stroke/TIA may also be affected by confounders such as differences in age, sex, the proportion of patients with ischemic stroke, hemorrhagic stroke or TIA (Table 1), the difference in inclusion criteria between studies and in the provider applying the scale. In most studies, the stroke scale assessment was performed by paramedics or nurses, making the evidence indirect for the first aid setting. However, Liferidge et al. found that lay providers were able to use the CPSS to detect stroke in volunteers with simulated stroke with $94.3 \%$ sensitivity (95\% CI [86.6-100.0]) and 82.93\% specificity (95\% CI [70.495.3]) [40]. Third, the overall Kappa for the review of titles/abstracts was moderate (Kappa $=0.44)$. This reflects difficulties in correctly identifying observational studies of stroke recognition in adults in a prehospital setting. However, based on a subsequent review of reference lists, we did not identify any additional articles that were missed during the review process. Finally, the risk of bias is serious or moderate in four of six studies due to possible confounding, missing data and the different time interval definitions for the outcome "time to treatment" $[20,29,33,34]$. Risk of bias is high or unclear in most of the diagnostic studies for patient selection or quality of the reference standard, and most of the studies failed to include all eligible consecutive participants. The methodology used by the studies is often different, measurement of the time to treatment is not the same, and the method and the length of training used to teach the score varied between studies. There is a high level of between-study heterogeneity, and therefore we must interpret the summary estimate result with caution.

\section{Conclusions}

The use of stroke recognition scales in the prehospital setting should be encouraged. They assist in the detection of the presence of stroke and reduce the time from symptom onset to definitive treatment. 


\section{Cureus}

scale to use remains complex. This review has shown that the use of the FAST and OPSS stroke recognition scales increases the proportion of stroke patients who receive therapy in the first hours following the onset of stroke. Furthermore, FAST and MASS are the scales with the highest sensitivity, while CPSS is the scale with the highest specificity. When blood glucose measurement is possible in the prehospital setting, LAPSS and MASS are scales with sensitivities similar to that for CPSS and FAST but provide greater specificity for the recognition of stroke.

\section{Appendices}

\section{APPENDIX A: Full search strategy for each database}

2015 ILCOR FATF CoSTR Systematic Review on Stroke Recognition

The results of the search strategy for the 2015 ILCOR First Aid Task Force (FATF) systematic review on stroke recognition are presented in Table 6.

\begin{tabular}{|c|c|c|}
\hline Databases Searched & Date of Search & Number of Articles \\
\hline All Medline <1946 - 2019> & September 26, 2019 & 2098 \\
\hline All Embase <1947 - 2019> & September 26, 2019 & 1316 \\
\hline \multirow[t]{2}{*}{ Cochrane Trials only $<1947-2019\rangle$} & September 28, 2019 & 30 \\
\hline & Total $(<1947-2019)$ & 3759 \\
\hline
\end{tabular}

TABLE 6: Results of the search for strategy, 2015 ILCOR FATF systematic review on stroke recognition

After duplicates were removed, title and abstract were screened, full-text articles were independently assessed and disagreements resolved through discussion, 24 articles were included in the 2015 systematic review.

Rerun Strategy from January 2014 to September 2019

The rerun strategy from January 2014 to September 2019 in three databases (MEDLINE, EMBASE and COCHRANE) and results are presented in Tables 7, 8 (Date of search: 26/09/2019).

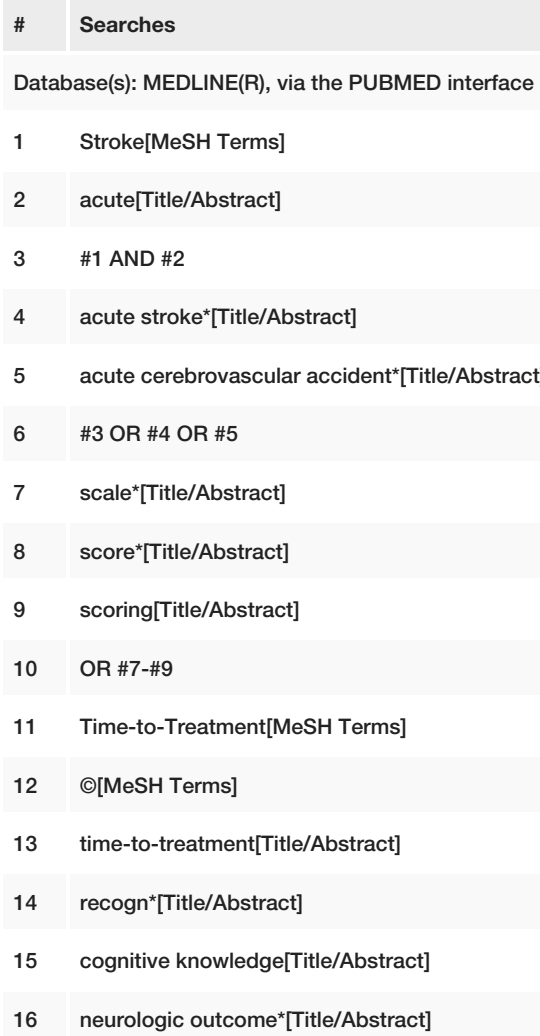




\section{Cureus}

\begin{tabular}{|c|c|c|}
\hline 17 & neurologic status[Title/Abstract] & 1837 \\
\hline 18 & OR \#11-\#17 & $1,875,735$ \\
\hline 19 & \#6 AND \#10 AND \#18 & 2185 \\
\hline 20 & animals[mh] NOT humans[mh] & $4,622,905$ \\
\hline 21 & "letter"[pt] OR "comment"[pt] OR "editorial"[pt] or Case Reports[ptyp] & $3,603,162$ \\
\hline 22 & \#19 NOT \#20 NOT \#21 & 2098 \\
\hline 23 & "2014/01/01"[PDAT] : "2019/09/26"[PDAT] & $6,592,744$ \\
\hline 24 & \#22 AND \#23 & 1042 \\
\hline \multicolumn{3}{|c|}{ Database(s): EMBASE (R), via Embase.com } \\
\hline 1 & 'cerebrovascular accident'/de & 308,474 \\
\hline 2 & acute:ab,ti & $1,585,164$ \\
\hline 3 & 1 AND 2 & 66,054 \\
\hline 4 & (acute near/3 stroke*):ab,ti & 54,551 \\
\hline 5 & 'acute cerebrovascular accident':ab,ti & 230 \\
\hline 6 & 'acute cerebrovascular accidents':ab,ti & 138 \\
\hline 7 & \#3 AND \#4 AND \#5 AND \#6 & 86,881 \\
\hline 8 & 'scoring system'/de & 246,463 \\
\hline 9 & 'rating scale'/de & 108,517 \\
\hline 10 & 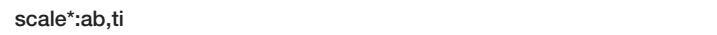 & $1,008,513$ \\
\hline 11 & score $^{*}: a b, t i$ & $1,340,709$ \\
\hline 12 & scoring:ab,ti & 117,138 \\
\hline 13 & OR \#8-\#12 & $2,138,593$ \\
\hline 14 & 'time to treatment'/de & 14,751 \\
\hline 15 & 'time factors'/de & 29,856 \\
\hline 16 & 'time to treatment':ab,ti & 6073 \\
\hline 17 & recogn*:ab,ti & 914,845 \\
\hline 18 & 'cognitive knowledge':ab,ti & 279 \\
\hline 19 & 'neurologic outcome':ab,ti & 3760 \\
\hline 20 & 'neurologic outcomes':ab,ti & 1836 \\
\hline 21 & 'neurologic status':ab,ti & 2500 \\
\hline 22 & OR \#14-\#21 & 968,595 \\
\hline 23 & \#7 AND \#13 AND \#22 & 1477 \\
\hline 24 & animal'/exp NOT 'human'/exp & $5,326,301$ \\
\hline 25 & \#23 NOT \#24 & 1460 \\
\hline 26 & [editorial]/lim OR [letter]/lim OR 'case report'/de & $4,003,436$ \\
\hline 27 & \#25 NOT \#26 & 1408 \\
\hline 28 & \#27 AND [embase]/lim & 1316 \\
\hline 29 & \#28 AND (2014-2019)/py & 775 \\
\hline \multicolumn{3}{|c|}{ Database(s): The Cochrane Library(R) } \\
\hline 1 & [mh Stroke] & 8451 \\
\hline 2 & acute:ab,ti & 122,671 \\
\hline 3 & \#1 AND \#2 & 2412 \\
\hline
\end{tabular}




\section{Cureus}

\begin{tabular}{|lll}
4 & (acute near/3 stroke*):ab,ti & 8484 \\
5 & "acute cerebrovascular accident"::ab,ti & 8 \\
6 & $\# 3$ OR \#4 OR \#5 & 9134 \\
7 & scale*:ab,ti & 150,761 \\
8 & score*:ab,ti & 220,166 \\
9 & scoring:ab,ti & 10,216 \\
10 & $\# 7$ OR \#8 OR \#9 & 298,259 \\
11 & [mh Time-to-Treatment] & 264 \\
12 & [mh "Time Factors"] & 63,000 \\
13 & "time-to-treatment":ab,ti & 1889 \\
14 & recogn*:ab,ti & 17,748 \\
15 & "cognitive knowledge":ab,ti & 33 \\
16 & "neurologic outcome*":ab,ti & 316 \\
17 & "neurologic status":ab,ti & 130 \\
18 & $\# 11$ OR \#12 OR \#13 OR \#14 OR \#15 OR \#16 OR \#17 & 82,461 \\
19 & $\# 6$ AND \#10 AND \#18 & 345 \\
& from Jan 2014 to Sep 2019 & 196
\end{tabular}

TABLE 7: Rerun strategy from January 2014 to September 2019 in MEDLINE, EMBASE and COCHRANE (date of search: 26/09/2019)

\begin{tabular}{|c|c|c|}
\hline Databases Searched & Date of Search & Number of Results \\
\hline All Medline <2014 - 2019> & September 26, 2019 & 1042 \\
\hline All Embase <2014 - 2019> & September 26, 2019 & 775 \\
\hline \multirow[t]{5}{*}{ Cochrane Trials only <2014 - 2019> } & September 28, 2019 & 196 \\
\hline & Total (<2014 - 2019) & 2013 \\
\hline & Total <2014 - 2019 (after deduplication) & 1651 \\
\hline & Total after title and abstract screen & 40 \\
\hline & Total after full text stage & 4 \\
\hline
\end{tabular}

TABLE 8: Result of the re-run of search strategy from 1 January 2014 to 26 September 2019

Rerun Strategy from September 2019 to May 2020

Results of the search strategy from September 2019 to May 2020 are presented in Tables 9, 10 (date of search 05/20/2020).

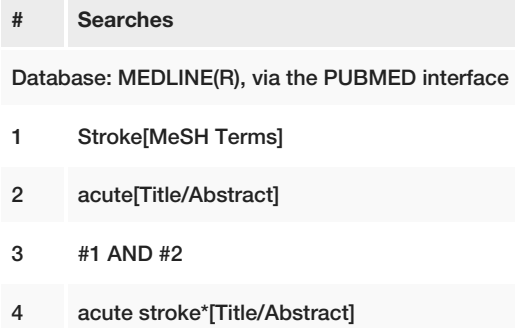




\section{Cureus}

\begin{tabular}{|c|c|c|}
\hline 5 & acute cerebrovascular accident ${ }^{*}[$ Title/Abstract] & 4 \\
\hline 6 & \#3 OR \#4 OR \#5 & 1351 \\
\hline 7 & scale*[Title/Abstract] & 53,910 \\
\hline 8 & score $^{*}[$ Title/Abstract] & 65,364 \\
\hline 9 & scoring[Title/Abstract] & 4852 \\
\hline 10 & OR \#7-\#9 & 104,908 \\
\hline 11 & Time-to-Treatment[MeSH Terms] & 342 \\
\hline 12 & "Time Factors" [MeSH Terms] & 5072 \\
\hline 13 & time-to-treatment[Title/Abstract] & 244 \\
\hline 14 & recogn ${ }^{\star}[$ Title/Abstract $]$ & 30,991 \\
\hline 15 & cognitive knowledge[Title/Abstract] & 10 \\
\hline 16 & neurologic outcome ${ }^{*}[$ Title/Abstract $]$ & 197 \\
\hline 17 & neurologic status[Title/Abstract] & 71 \\
\hline 18 & OR \#11-\#17 & 36,660 \\
\hline 19 & \#6 AND \#10 AND \#18 & 65 \\
\hline 20 & animals[mh] NOT humans[mh] & 27,043 \\
\hline 21 & "letter"[pt] OR "comment"[pt] OR "editorial"[pt] or Case Reports[ptyp] & 88,312 \\
\hline 22 & \#19 NOT \#20 NOT \#21 & 65 \\
\hline 23 & "2014/01/01"[PDAT] : "2019/09/26"[PDAT] & \\
\hline 24 & \#22 AND \#23 & 65 \\
\hline \multicolumn{3}{|c|}{ Database(s): EMBASE (R), via Embase.com } \\
\hline 1 & 'cerebrovascular accident'/de & 19,122 \\
\hline 2 & acute:ab,ti & 64,060 \\
\hline 3 & 1 AND 2 & 4529 \\
\hline 4 & (acute near/3 stroke*):ab,ti & 2787 \\
\hline 5 & 'acute cerebrovascular accident':ab,ti & 19 \\
\hline 6 & 'acute cerebrovascular accidents':ab,ti & 5 \\
\hline 7 & \#3 OR \#4 OR \#5 OR \#6 & 5234 \\
\hline 8 & 'scoring system'/de & 10,010 \\
\hline 9 & 'rating scale'/de & 2474 \\
\hline 10 & scale $^{*}: a b, t i$ & 67,006 \\
\hline 11 & score $^{*}: a b, t i$ & 86,475 \\
\hline 12 & scoring:ab,ti & 6542 \\
\hline 13 & OR \#8-\#12 & 137,402 \\
\hline 14 & 'time to treatment'/de & 1767 \\
\hline 15 & 'time factors'/de & 3193 \\
\hline 16 & 'time to treatment':ab,ti & 467 \\
\hline 17 & recogn*:ab,ti & 37,759 \\
\hline 18 & 'cognitive knowledge':ab,ti & 7 \\
\hline 19 & 'neurologic outcome':ab,ti & 159 \\
\hline 20 & 'neurologic outcomes':ab,ti & 153 \\
\hline 21 & 'neurologic status':ab,ti & 86 \\
\hline
\end{tabular}




\section{Cureus}

\begin{tabular}{|c|c|c|}
\hline 22 & OR \#14-\#21 & 42,985 \\
\hline 23 & \#7 AND \#13 AND \#22 & 97 \\
\hline 24 & 'animal'/exp NOT 'human'/exp & 111,646 \\
\hline 25 & \#23 NOT \#24 & 96 \\
\hline 26 & [editorial]/lim OR [letter]/lim OR 'case report'/de & 134,589 \\
\hline 27 & \#25 NOT \#26 & 86 \\
\hline 28 & \#27 AND [embase]/lim & 75 \\
\hline 29 & \#28 AND (2014-2019)/py (from 27/09/2019 to $25 / 05 / 2020$ for rerun 2020) & 93 \\
\hline \multicolumn{3}{|c|}{ Database(s): The Cochrane Library(R) } \\
\hline 1 & [mh Stroke] & 918 \\
\hline 2 & acute:ab,ti & 3664 \\
\hline 3 & \#1 AND \#2 & 316 \\
\hline 4 & (acute near/3 stroke $\left.{ }^{\star}\right): a b, t i$ & 105 \\
\hline 5 & "acute cerebrovascular accident ${ }^{\star "}: \mathrm{ab}, \mathrm{ti}$ & 0 \\
\hline 6 & \#3 OR \#4 OR \#5 & 173 \\
\hline 7 & scale*:ab,ti & 9795 \\
\hline 8 & score*:ab,ti & 10,794 \\
\hline 9 & scoring:ab,ti & 152 \\
\hline 10 & \#7 OR \#8 OR \#9 & 15,526 \\
\hline 11 & [mh Time-to-Treatment] & 44 \\
\hline 12 & [mh "Time Factors"] & 746 \\
\hline 13 & "time-to-treatment":ab,ti & 95 \\
\hline 14 & recogn*:ab,ti & 1890 \\
\hline 15 & "cognitive knowledge":ab,ti & 2 \\
\hline 16 & "neurologic outcome*":ab,ti & 0 \\
\hline 17 & "neurologic status":ab,ti & 0 \\
\hline 18 & \#11 OR \#12 OR \#13 OR \#14 OR \#15 OR \#16 OR \#17 & 643 \\
\hline \multirow[t]{2}{*}{19} & \#6 AND \#10 AND \#18 & 21 \\
\hline & from September 2019 to May 2020 & 23 \\
\hline
\end{tabular}

TABLE 9: Results of the search strategy from September 2019 to May 2020 (date of search 05/20/2020) 


\section{Cureus}

\begin{tabular}{|c|c|c|}
\hline Databases Searched & Date of Search & Number of Articles \\
\hline All Medline & May $25,2020,2019$ & 65 \\
\hline All Embase < Sept. 2019 to May 2020 > & May 25, 2020, 2019 & 94 \\
\hline \multirow[t]{5}{*}{ Cochrane Trials only < Sept. 2019 to May 2020> } & May $25,2020,2019$ & 22 \\
\hline & Total (Sept. 2019 to May 2020) & 181 \\
\hline & Total < Sept. 2019 to May 2020 (after deduplication) & 163 \\
\hline & Total after title and abstract screen & 6 \\
\hline & Total after full text stage & 0 \\
\hline
\end{tabular}

TABLE 10: Results of the rerun search strategy from 26 September 2019 to 25 May 2020

Global Search Strategy from Inception to May 2020

The results of the global search strategy are presented in Table 11.

\begin{tabular}{|l|l|l|}
\hline Sources & Number of Articles & Number of Articles Selected \\
\hline 2015 ILCOR FATF systematic review on stroke recognition & 24 & 46 \\
\hline Other sources & 8 & 4 \\
\hline 2019 rerun search strategy ILCOR FATF & 1651 & 0 \\
\hline 2020 rerun search strategy ILCOR FATF & 163 & 24 \\
\hline
\end{tabular}

TABLE 11: Result of the global search strategy for stroke recognition

APPENDIX B: Characteristics of excluded studies

The characteristics of excluded studies are presented in Table 12 .

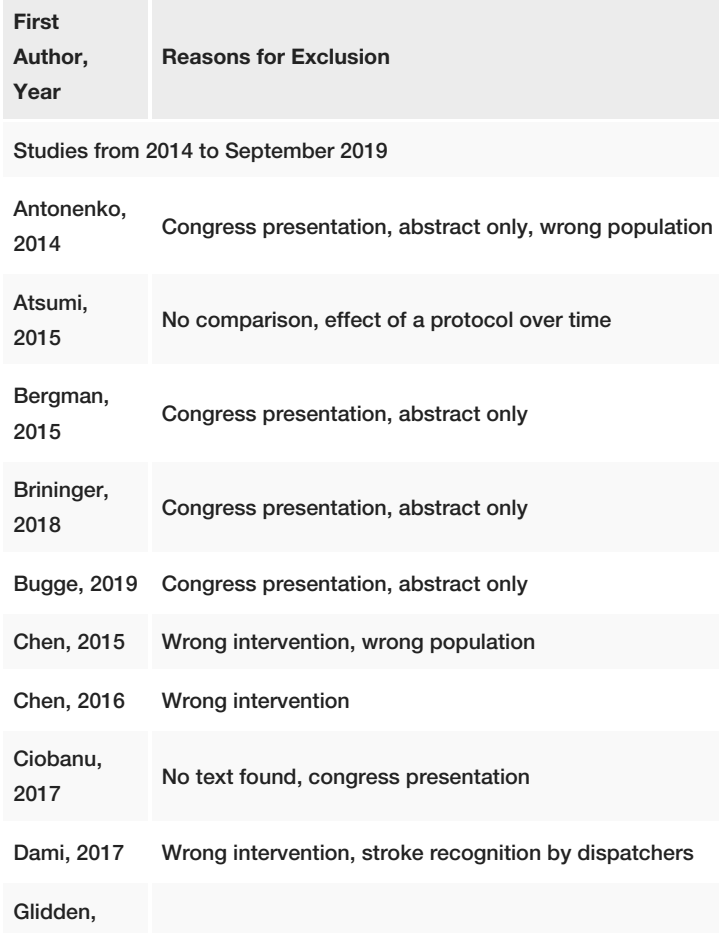




\section{Cureus}

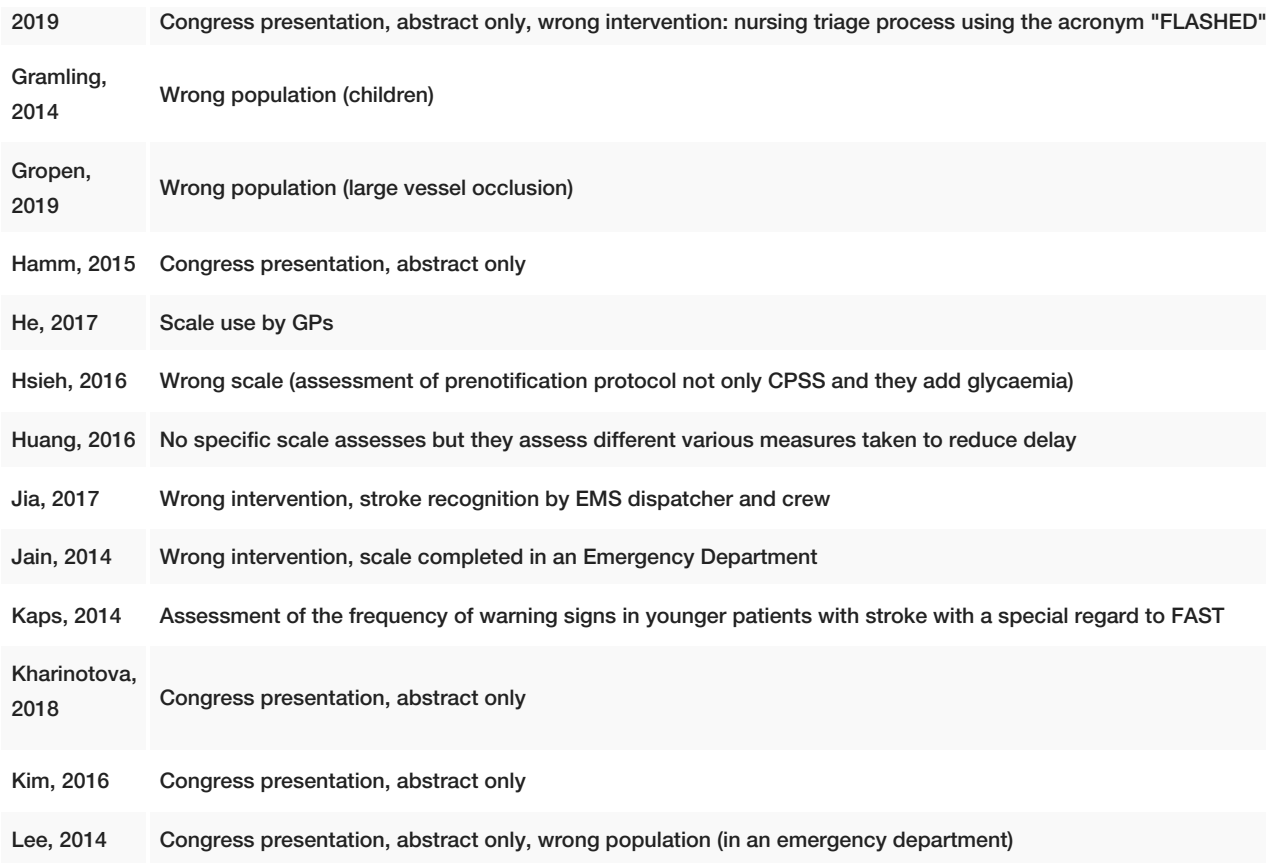




\section{Cureus}

\begin{tabular}{|c|c|}
\hline $\begin{array}{l}\text { Kleindorfer, } \\
2007\end{array}$ & Exclusion (retrospectively collection of signs by nurses in medical records of all stroke patients) \\
\hline You, 2013 & $\begin{array}{l}\text { Wrong population (thrombolytic candidates in acute ischemic stroke only). Exclusion (the aim of this study is to investigate the } \\
\text { usefulness of the CPSS to determine stroke severity by comparing CPSS and NIHSS scores in patients who may be candidates for } \\
\text { thrombolysis on arrival at the hospital within } 6 \text { hours of symptom onset) }\end{array}$ \\
\hline Nazliel, 2008 & $\begin{array}{l}\text { Wrong population (the aim of the study is to determine whether LAMS scores can predict the presence of large vessel occlusions } \\
\text { in acute cerebral ischemia patients) }\end{array}$ \\
\hline Nor, 2005 & $\begin{array}{l}\text { Wrong intervention (emergency physicians with ROSIER and retrospective calculation based on neurologist-recorded signs for } \\
\text { CPSS, LAPSS and FAST) }\end{array}$ \\
\hline $\begin{array}{l}\text { Whiteley, } \\
2011\end{array}$ & Wrong intervention (The stroke scale was completed by emergency physicians) \\
\hline $\begin{array}{l}\text { Yock- } \\
\text { Corrales, } \\
2011\end{array}$ & Wrong population and exclusion criteria (scale applied retrospectively to children only with ischemic stroke) \\
\hline \multicolumn{2}{|c|}{ Studies excluded from rerun search strategy from 2019 to 2020} \\
\hline Kaps, 2014 & Wrong population (younger people), wrong intervention (retrospective analysis on hospital signs) \\
\hline $\begin{array}{l}\text { Willaert, } \\
2020\end{array}$ & Congress presentation, abstract only \\
\hline Colton, 2020 & Wrong intervention, wrong population (Intracranial hemorrhage only) \\
\hline Car, 2020 & Wrong intervention (emergent large vessel occlusion) \\
\hline $\begin{array}{l}\text { Madhok, } \\
2019\end{array}$ & Wrong intervention (no limit to CPSS but a whole protocol before and after the implementation) \\
\hline Lee, 2020 & Wrong intervention \\
\hline
\end{tabular}

\section{TABLE 12: Characteristics of excluded studies}

APPENDIX C: Evidence profile tables

Evidence Profile Tables for Observational Studies

Evidence profile tables for observational studies are presented in Tables 13-18. 


\section{Cureus}

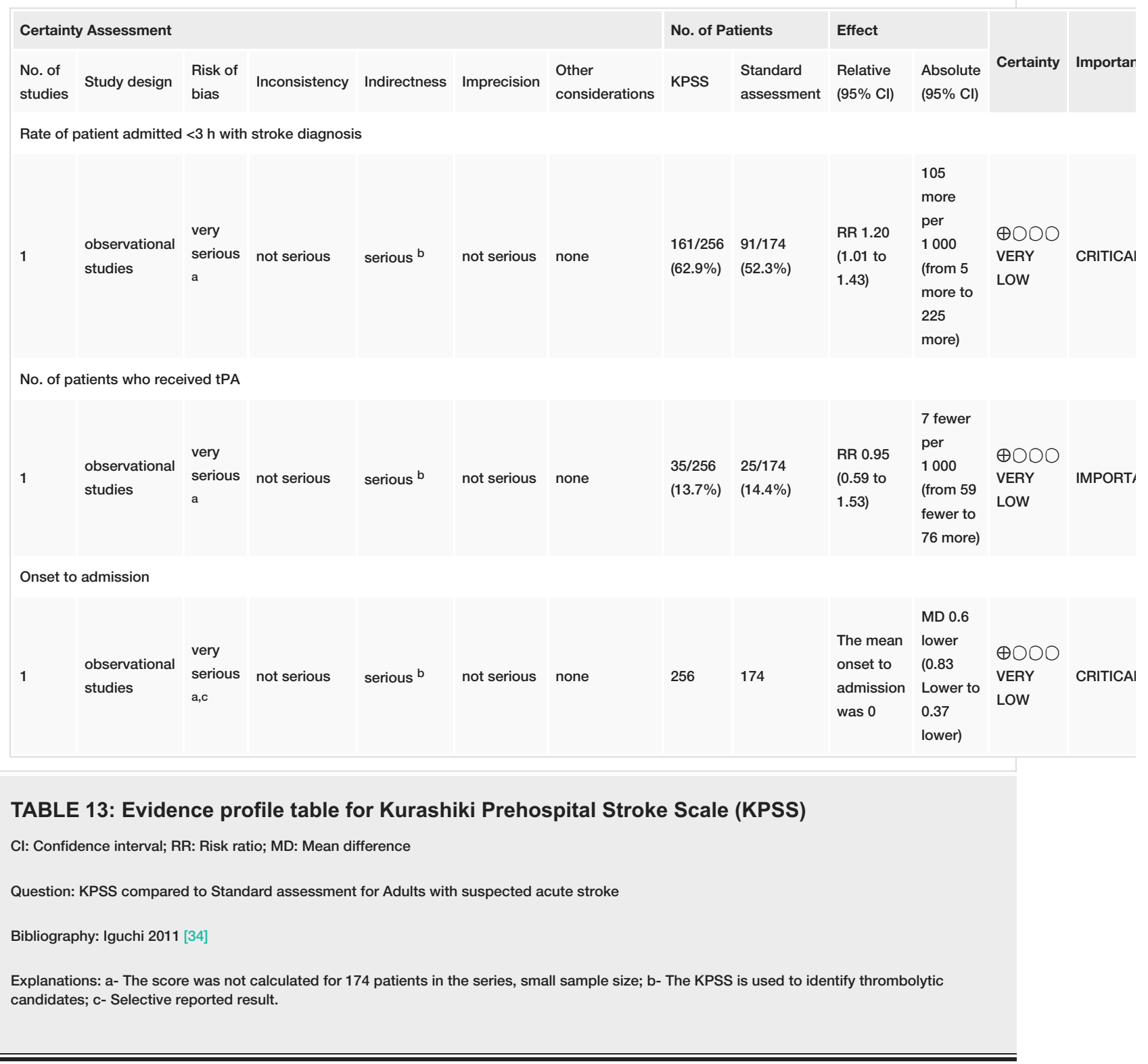




\section{Cureus}

\begin{tabular}{|c|c|c|c|c|c|c|c|c|c|c|c|c|}
\hline \multicolumn{7}{|c|}{ Certainty Assessment } & \multicolumn{2}{|c|}{ No. of Patients } & \multicolumn{2}{|l|}{ Effect } & \multirow[b]{2}{*}{ Certainty } & \multirow[b]{2}{*}{ Importance } \\
\hline $\begin{array}{l}\text { No. of } \\
\text { studies }\end{array}$ & Study design & $\begin{array}{l}\text { Risk of } \\
\text { bias }\end{array}$ & Inconsistency & Indirectness & Imprecision & $\begin{array}{l}\text { Other } \\
\text { considerations }\end{array}$ & LAPSS & $\begin{array}{l}\text { Standard } \\
\text { assessment }\end{array}$ & $\begin{array}{l}\text { Relative } \\
\text { (95\% } \\
\text { Cl) }\end{array}$ & $\begin{array}{l}\text { Absolute } \\
(95 \% \mathrm{Cl})\end{array}$ & & \\
\hline \multicolumn{13}{|c|}{ Rate onset to admission $<2 \mathrm{~h}$} \\
\hline 1 & $\begin{array}{l}\text { observational } \\
\text { studies }\end{array}$ & $\begin{array}{l}\text { serious } \\
\text { a }\end{array}$ & not serious & serious $^{b}$ & not serious & none & $\begin{array}{l}418 / 6 / 4 \\
(62.0 \%)\end{array}$ & $\begin{array}{l}210 / 362 \\
(58.0 \%)\end{array}$ & $\begin{array}{l}\text { RR } 1.07 \\
(0.96 \text { to } \\
1.19)\end{array}$ & $\begin{array}{l}41 \text { more } \\
\text { per } \\
1000 \\
\text { (from } 23 \\
\text { fewer to } \\
110 \\
\text { more) }\end{array}$ & $\begin{array}{l}\oplus 000 \\
\text { VERY } \\
\text { LOW }\end{array}$ & CRITICAL \\
\hline \multicolumn{13}{|c|}{ Onset to ED Arrival } \\
\hline 1 & $\begin{array}{l}\text { observational } \\
\text { studies }\end{array}$ & $\begin{array}{l}\text { serious } \\
\text { a }\end{array}$ & not serious & serious $^{b}$ & not serious & none & 680 & 359 & - & $\begin{array}{l}\text { MD } 132 \\
\text { higher } \\
\text { (14.68 } \\
\text { higher to } \\
249.32 \\
\text { higher) }\end{array}$ & $\begin{array}{l}\oplus 000 \\
\text { VERY } \\
\text { LOW }\end{array}$ & CRITICAL \\
\hline \multicolumn{13}{|c|}{ Treatment with IV tPA of confirmed stroke cases } \\
\hline 1 & $\begin{array}{l}\text { observational } \\
\text { studies }\end{array}$ & $\begin{array}{l}\text { serious } \\
\text { a }\end{array}$ & not serious & serious $^{b}$ & not serious & none & $\begin{array}{l}64 / 533 \\
(12.0 \%)\end{array}$ & $\begin{array}{l}21 / 198 \\
(10.6 \%)\end{array}$ & $\begin{array}{l}\text { RR } 1.13 \\
(0.71 \text { to } \\
1.80)\end{array}$ & $\begin{array}{l}14 \text { more } \\
\text { per } \\
1000 \\
\text { (from } 31 \\
\text { fewer to } \\
85 \text { more) }\end{array}$ & $\begin{array}{l}\text { ๑OOO } \\
\text { VERY } \\
\text { LOW }\end{array}$ & IMPORTAN \\
\hline \multicolumn{13}{|c|}{$\mathrm{Nb}$ of good diagnosis by paramedics at discharge } \\
\hline 1 & $\begin{array}{l}\text { observational } \\
\text { studies }\end{array}$ & $\begin{array}{l}\text { serious } \\
\text { a }\end{array}$ & not serious & serious $^{b}$ & not serious & none & $\begin{array}{l}709 / 895 \\
(79.2 \%)\end{array}$ & $\begin{array}{l}198 / 323 \\
(61.3 \%)\end{array}$ & $\begin{array}{l}\text { RR } 1.29 \\
(1.18 \text { to } \\
1.42)\end{array}$ & $\begin{array}{l}178 \\
\text { more } \\
\text { per } \\
1000 \\
\text { (from } \\
110 \\
\text { more to } \\
257 \\
\text { more) }\end{array}$ & $\begin{array}{l}\text { ๑OOO } \\
\text { VERY } \\
\text { LOW }\end{array}$ & IMPORTAN \\
\hline
\end{tabular}

\section{TABLE 14: Evidence profile table for Los Angeles Prehospital Stroke Scale (LAPSS)}

Cl: Confidence interval; RR: Risk ratio; MD: Mean difference; OR: Odds ratio; IV TPA: Intravenous tissue Plasminogen Activator

Question: LAPSS compared to Standard assessment for Adults with suspected acute stroke

Bibliography: Wojner-Alexandrov, 2005 [24]

Explanations: a- Downgrade for serious risk of bias for selection of participants; b- The LAPSS is used for paramedics' decision. The assessment is not limited only to the LAPSS. 


\section{Cureus}

\begin{tabular}{|c|c|c|c|c|c|c|c|c|c|c|c|c|}
\hline \multicolumn{7}{|c|}{ Certainty Assessment } & \multicolumn{2}{|c|}{ No. of Patients } & \multicolumn{2}{|l|}{ Effect } & \multirow[b]{2}{*}{ Certainty } & \multirow[b]{2}{*}{ Importanc } \\
\hline $\begin{array}{l}\text { No. of } \\
\text { studies }\end{array}$ & Study design & $\begin{array}{l}\text { RiSk of } \\
\text { bias }\end{array}$ & Inconsistency & Indirectness & Imprecision & $\begin{array}{l}\text { Other } \\
\text { considerations }\end{array}$ & OPSS & $\begin{array}{l}\text { Standard } \\
\text { assessment }\end{array}$ & $\begin{array}{l}\text { Relative } \\
\text { (95\% } \\
\mathrm{Cl)}\end{array}$ & $\begin{array}{l}\text { Absolute } \\
(95 \% \mathrm{Cl})\end{array}$ & & \\
\hline \multicolumn{13}{|c|}{ Ischemic stroke patients arriving $<3$ hours } \\
\hline 1 & $\begin{array}{l}\text { observational } \\
\text { studies }\end{array}$ & $\begin{array}{l}\text { very } \\
\text { serious } \\
\text { a }\end{array}$ & not serious & not serious & not serious & none & $\begin{array}{l}1 / 8 / 554 \\
(32.1 \%)\end{array}$ & $\begin{array}{l}69 / 307 \\
(22.5 \%)\end{array}$ & $\begin{array}{l}\text { RR } 1.43 \\
\text { (1.12 to } \\
1.82)\end{array}$ & $\begin{array}{l}\text { per } \\
1000 \\
\text { (from } 27 \\
\text { more to } \\
184 \\
\text { more) }\end{array}$ & $\begin{array}{l}\oplus 000 \\
\text { VERY } \\
\text { LOW }\end{array}$ & CRITICAL \\
\hline \multicolumn{13}{|c|}{ Rate of tPA administration (all patients) } \\
\hline 1 & $\begin{array}{l}\text { observational } \\
\text { studies }\end{array}$ & $\begin{array}{l}\text { very } \\
\text { serious } \\
\text { a }\end{array}$ & not serious & not serious & not serious & none & $\begin{array}{l}56 / 554 \\
(10.1 \%)\end{array}$ & $\begin{array}{l}18 / 307 \\
(5.9 \%)\end{array}$ & $\begin{array}{l}\text { RR } 1.72 \\
\text { (1.03 to } \\
2.88)\end{array}$ & $\begin{array}{l}42 \text { more } \\
\text { per } \\
1000 \\
\text { (from } 2 \\
\text { more to } \\
110 \\
\text { more) }\end{array}$ & $\begin{array}{l}\oplus 000 \\
\text { VERY } \\
\text { LOW }\end{array}$ & IMPORTAN \\
\hline \multicolumn{13}{|c|}{ Dlagnosis Ischemic stroke } \\
\hline 1 & $\begin{array}{l}\text { observational } \\
\text { studies }\end{array}$ & $\begin{array}{l}\text { very } \\
\text { serious } \\
\text { a }\end{array}$ & not serious & not serious & not serious & none & $\begin{array}{l}290 / 554 \\
(52.3 \%)\end{array}$ & $\begin{array}{l}145 / 307 \\
(47.2 \%)\end{array}$ & $\begin{array}{l}\text { RR } 1,11 \\
(0.96 \text { to } \\
1.28)\end{array}$ & 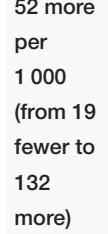 & $\begin{array}{l}\oplus 000 \\
\text { VERY } \\
\text { LOW }\end{array}$ & IMPORTAN \\
\hline
\end{tabular}

\section{TABLE 15: Evidence profile table for Ontario Prehospital Stroke Scale (OPSS)}

Cl: Confidence interval; RR: Risk ratio; OR: Odds ratio; IV TPA: Intravenous tissue Plasminogen Activator

Question: OPSST compared with standard assessment for adults with suspected acute stroke

Bibliography: Chenkin, 2009 [29]

Explanations: a- Very serious risk of bias due to deviation from intended interventions, missing data and confounding factor 


\section{Cureus}

\begin{tabular}{|c|c|c|c|c|c|c|c|c|c|c|c|c|}
\hline \multicolumn{7}{|c|}{ Certainty Assessment } & \multicolumn{2}{|c|}{ No. of Patients } & \multicolumn{2}{|l|}{ Effect } & \multirow[b]{2}{*}{ Certainty } & \multirow[b]{2}{*}{ Importanc } \\
\hline $\begin{array}{l}\text { No. of } \\
\text { studies }\end{array}$ & Study design & $\begin{array}{l}\text { Risk of } \\
\text { bias }\end{array}$ & Inconsistency & Indirectness & Imprecision & $\begin{array}{l}\text { Other } \\
\text { considerations }\end{array}$ & FASTER & $\begin{array}{l}\text { Standard } \\
\text { assessment }\end{array}$ & $\begin{array}{l}\text { Relative } \\
\text { (95\% } \\
\mathrm{Cl})\end{array}$ & $\begin{array}{l}\text { Absolute } \\
(95 \% \mathrm{Cl})\end{array}$ & & \\
\hline \multicolumn{13}{|c|}{ Symptom onset to treatment time } \\
\hline 1 & $\begin{array}{l}\text { observational } \\
\text { studies }\end{array}$ & $\begin{array}{l}\text { very } \\
\text { serious } \\
\text { a }\end{array}$ & not serious & not serious & serious $^{b}$ & none & 17 & (2. & 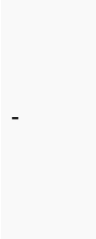 & $\begin{array}{l}\text { MD } 32 \\
\text { min } \\
\text { fewer } \\
\text { (53 } \\
\text { fewer to } \\
11 \\
\text { fewer) }\end{array}$ & $\begin{array}{l}\text { 円OOO } \\
\text { VERY } \\
\text { LOW }\end{array}$ & CRITICAL \\
\hline \multicolumn{13}{|c|}{ Door to CT time } \\
\hline 1 & $\begin{array}{l}\text { observational } \\
\text { studies }\end{array}$ & $\begin{array}{l}\text { very } \\
\text { serious } \\
\text { a }\end{array}$ & not serious & not serious & serious $^{b}$ & none & 17 & 17 & - & $\begin{array}{l}\text { MD } 30 \\
\text { min } \\
\text { fewer } \\
\text { (50 } \\
\text { fewer to } \\
11 \\
\text { fewer) }\end{array}$ & $\begin{array}{l}\oplus O O O \\
\text { VERY } \\
\text { LOW }\end{array}$ & CRITICAL \\
\hline \multicolumn{13}{|c|}{ Door to needle time } \\
\hline 1 & $\begin{array}{l}\text { observational } \\
\text { studies }\end{array}$ & $\begin{array}{l}\text { very } \\
\text { serious } \\
\text { a }\end{array}$ & not serious & not serious & serious $^{\mathrm{b}}$ & none & 17 & 17 & - & $\begin{array}{l}\text { MD } 46 \\
\text { min } \\
\text { fewer } \\
\text { (71 } \\
\text { fewer to } \\
21 \\
\text { fewer) }\end{array}$ & $\begin{array}{l}\text { 円OOO } \\
\text { VERY } \\
\text { LOW }\end{array}$ & CRITICAL \\
\hline \multicolumn{13}{|c|}{ Onset to door } \\
\hline 1 & $\begin{array}{l}\text { observational } \\
\text { studies }\end{array}$ & $\begin{array}{l}\text { very } \\
\text { serious } \\
\text { a }\end{array}$ & not serious & not serious & serious $^{b}$ & none & 17 & 17 & - & $\begin{array}{l}\text { MD } 17 \\
\text { min } \\
\text { more } \\
(7.3 \\
\text { fewer to } \\
41 \text { more } \\
\text { ) }\end{array}$ & $\begin{array}{l}\text { ๑OOO } \\
\text { VERY } \\
\text { LOW }\end{array}$ & CRITICAL \\
\hline \multicolumn{13}{|c|}{ Rate of thrombolytic therapy } \\
\hline 1 & $\begin{array}{l}\text { observational } \\
\text { studies }\end{array}$ & $\begin{array}{l}\text { very } \\
\text { serious } \\
\text { a }\end{array}$ & not serious & not serious & serious $^{\mathrm{b}}$ & none & $\begin{array}{l}22 / 115 \\
(19.1 \%)\end{array}$ & $5 / 67(7.5 \%)$ & $\begin{array}{l}\text { RR } 2.56 \\
(1.02 \text { to } \\
6.45)\end{array}$ & $\begin{array}{l}116 \\
\text { more } \\
\text { per } \\
1000 \\
\text { (from } 1 \\
\text { more to } \\
407 \\
\text { more) }\end{array}$ & $\begin{array}{l}\oplus O O O \\
\text { VERY } \\
\text { LOW }\end{array}$ & IMPORTAA \\
\hline
\end{tabular}

TABLE 16: Evidence profile table for Face, Arm, Speech Time, Emergency Response Protocol (FASTER)

Cl: Confidence interval; MD: Mean difference; RR: Risk ratio

Explanations: a- Very serious risk of bias due to confounding and selection for the reported result; b- Serious imprecision due to incomplete data reporting

Question: FASTER compared to Standard assessment for Adults with suspected acute stroke

Bibliography: O’Brien, 2012 [20] 


\section{Cureus}

\begin{tabular}{|c|c|c|c|c|c|c|c|c|c|c|c|c|}
\hline \multicolumn{7}{|c|}{ Certainty Assessment } & \multicolumn{2}{|c|}{ No. of Patients } & \multicolumn{2}{|l|}{ Effect } & \multirow[b]{2}{*}{ Certainty } & \multirow[b]{2}{*}{ Importance } \\
\hline $\begin{array}{l}\text { No. of } \\
\text { studies }\end{array}$ & Study design & $\begin{array}{l}\text { Risk of } \\
\text { bias }\end{array}$ & Inconsistency & Indirectness & Imprecision & $\begin{array}{l}\text { Other } \\
\text { considerations }\end{array}$ & FAST & $\begin{array}{l}\text { Standard } \\
\text { assessment }\end{array}$ & $\begin{array}{l}\text { Relative } \\
\text { (95\% } \\
\text { Cl) }\end{array}$ & $\begin{array}{l}\text { Absolute } \\
(95 \% \mathrm{Cl})\end{array}$ & & \\
\hline \multicolumn{13}{|c|}{ Rate of patients admitted $<3 \mathrm{~h}$ with stroke diagnosis } \\
\hline 1 & $\begin{array}{l}\text { observational } \\
\text { studies }\end{array}$ & $\begin{array}{l}\text { very } \\
\text { serious } \\
\text { a }\end{array}$ & not serious & not serious & not serious & none & $\begin{array}{l}66 / 137 \\
(48.2 \%)\end{array}$ & $\begin{array}{l}32 / 219 \\
(14.6 \%)\end{array}$ & $\begin{array}{l}\text { RR } 3.30 \\
(2.29 \text { to } \\
4.75)\end{array}$ & $\begin{array}{l}336 \\
\text { more } \\
\text { per } \\
1000 \\
\text { (from } \\
188 \\
\text { more to } \\
548 \\
\text { more) }\end{array}$ & $\begin{array}{l}\text { ๑OOO } \\
\text { VERY } \\
\text { LOW }\end{array}$ & CRITICAL \\
\hline
\end{tabular}

\section{TABLE 17: Evidence profile table for Face, Arm, Speech, Time to Call (FAST) Scale}

Cl: Confidence interval; RR: Risk ratio

Question: FAST compared to Standard assessment for Adults with suspected acute stroke

Bibliography: Harbison, 2003 [33]

Explanations: a- Fast is integrated in a specific protocol call "rapid ambulance protocol" and compared with PCDs and ED doctor's diagnosis of stroke. No information about confounding factors.

\begin{tabular}{|c|c|c|c|c|c|c|c|c|c|c|c|c|}
\hline \multicolumn{7}{|c|}{ Certainty Assessment } & \multicolumn{2}{|c|}{ No. of Patients } & \multicolumn{2}{|l|}{ Effect } & \multirow[b]{2}{*}{ Certainty } & \multirow[b]{2}{*}{ Importan } \\
\hline $\begin{array}{l}\text { No. of } \\
\text { studies }\end{array}$ & Study design & $\begin{array}{l}\text { Risk of } \\
\text { bias }\end{array}$ & Inconsistency & Indirectness & Imprecision & $\begin{array}{l}\text { Other } \\
\text { considerations }\end{array}$ & $\begin{array}{l}\text { Knowledge } \\
\text { on FAST } \\
\text { symptoms }\end{array}$ & Placebo & $\begin{array}{l}\text { Relative } \\
\text { (95\% } \\
\text { Cl) }\end{array}$ & $\begin{array}{l}\text { Absolute } \\
(95 \% \text { Cl) }\end{array}$ & & \\
\hline
\end{tabular}

Before education and immediately after

\begin{tabular}{|c|c|c|c|c|c|c|c|c|c|c|c|}
\hline 1 & $\begin{array}{l}\text { observational } \\
\text { studies }\end{array}$ & $\begin{array}{l}\text { not } \\
\text { serious }\end{array}$ & not serious & not serious & serious $^{\mathrm{a}}$ & none & $\begin{array}{l}68 / 72 \\
(94.4 \%)\end{array}$ & $\begin{array}{l}55 / 72 \\
(76.4 \%)\end{array}$ & $\begin{array}{l}\text { RR } 1.24 \\
(1.07 \text { to } \\
1.42)\end{array}$ & $\begin{array}{l}183 \\
\text { more } \\
\text { per } \\
1000 \\
\text { (from } 53 \\
\text { more to } \\
213 \\
\text { more) }\end{array}$ & $\begin{array}{l}\oplus \oplus \oplus \bigcirc \\
\text { MODERATE }\end{array}$ \\
\hline
\end{tabular}

After education and 3 months after

\begin{tabular}{|c|c|c|c|c|c|c|c|c|c|c|c|}
\hline 1 & $\begin{array}{l}\text { observational } \\
\text { studies }\end{array}$ & $\begin{array}{l}\text { not } \\
\text { serious }\end{array}$ & not serious & not serious & serious $^{a}$ & none & $\begin{array}{l}63 / 65 \\
(96.9 \%)\end{array}$ & $\begin{array}{l}64 / 65 \\
(98.5 \%)\end{array}$ & $\begin{array}{l}\text { RR } 0.98 \\
(0.93 \text { to } \\
1.04)\end{array}$ & $\begin{array}{l}20 \text { fewer } \\
\text { per } \\
1000 \\
\text { (from } 69 \\
\text { fewer to } \\
39 \text { more) }\end{array}$ & $\begin{array}{l}\oplus \oplus \oplus \bigcirc \\
\text { MODERATE }\end{array}$ \\
\hline
\end{tabular}

\section{TABLE 18: Evidence profile table for increased public/layperson recognition of signs of stroke}

Cl: Confidence interval; RR: Risk ratio; OR: Odds ratio

Question: Knowledge on FAST symptoms Pretest and Posttest Survey Results

Bibliography: Wall, 2008 [23]

Explanations: a- Only one study is about cognitive knowledge. This research identifies messages with evidence-based effectiveness for communicating stroke signs and symptoms. The population is limited to non-Hispanic white and non-Hispanic black women aged 40 to 64 years.

Evidence Profile Tables for Diagnosis Studies 


\section{Cureus}

Evidence profile tables for diagnosis studies are presented in Tables 19-22.

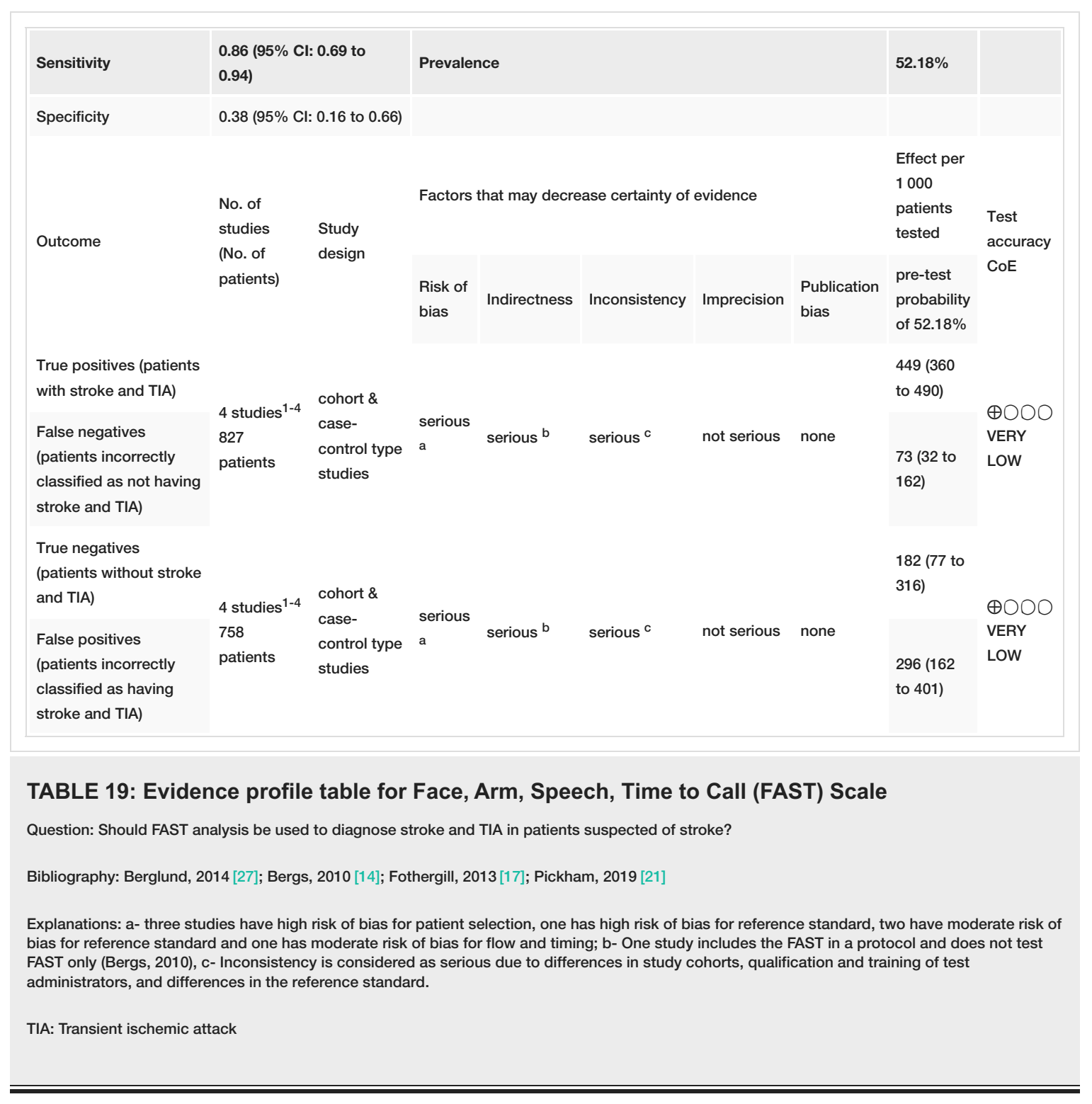




\section{Cureus}

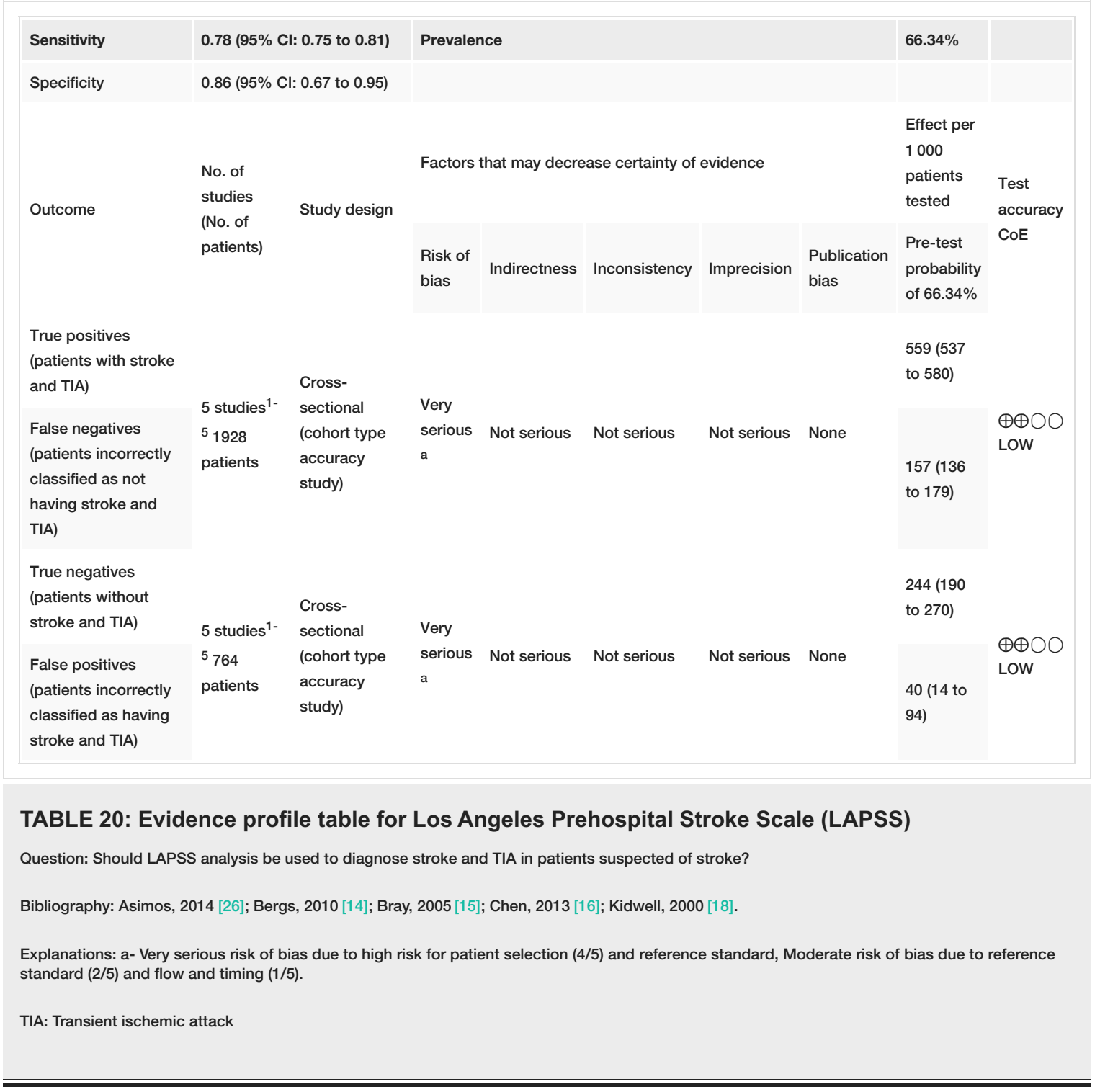




\section{Cureus}

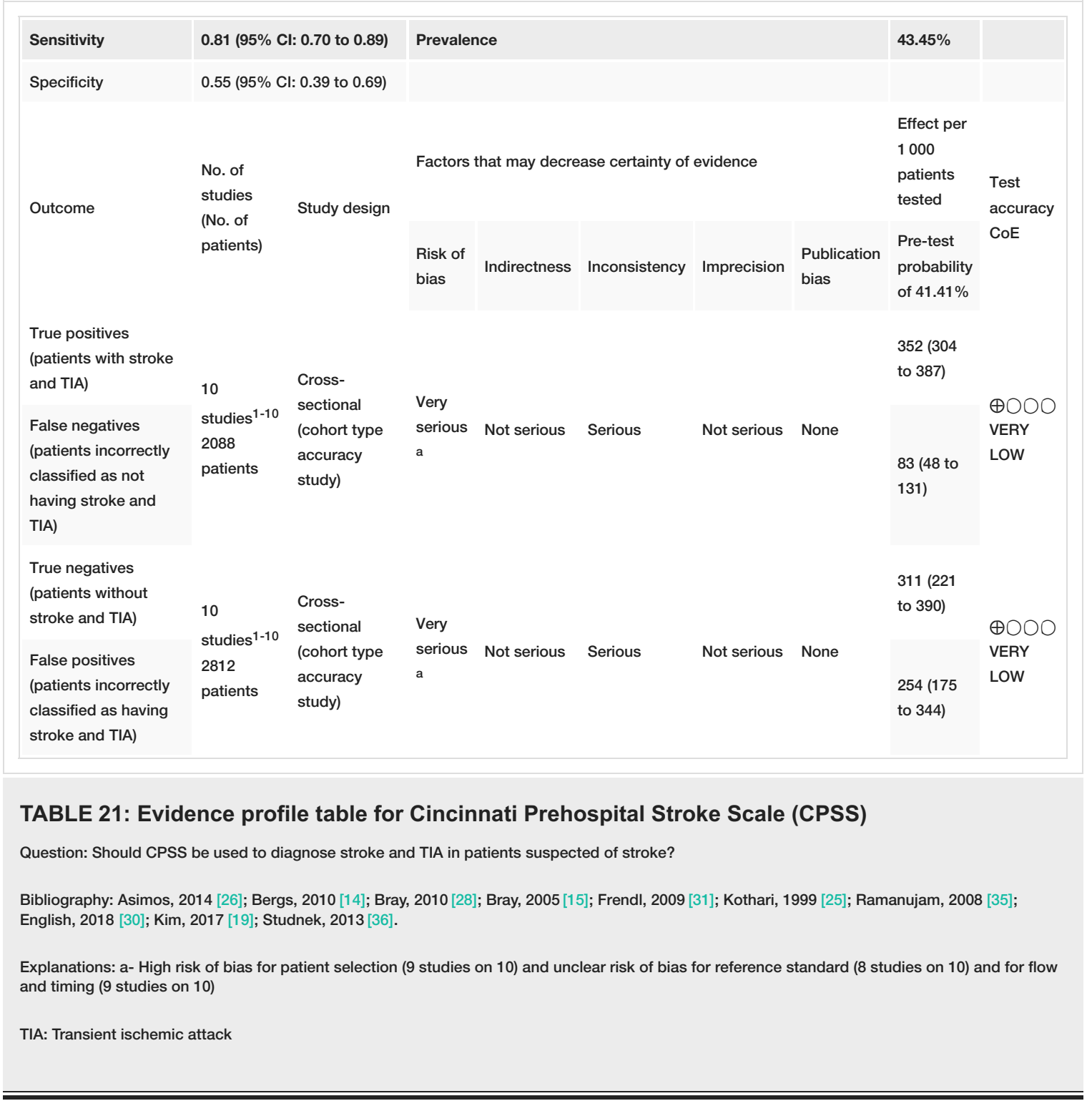




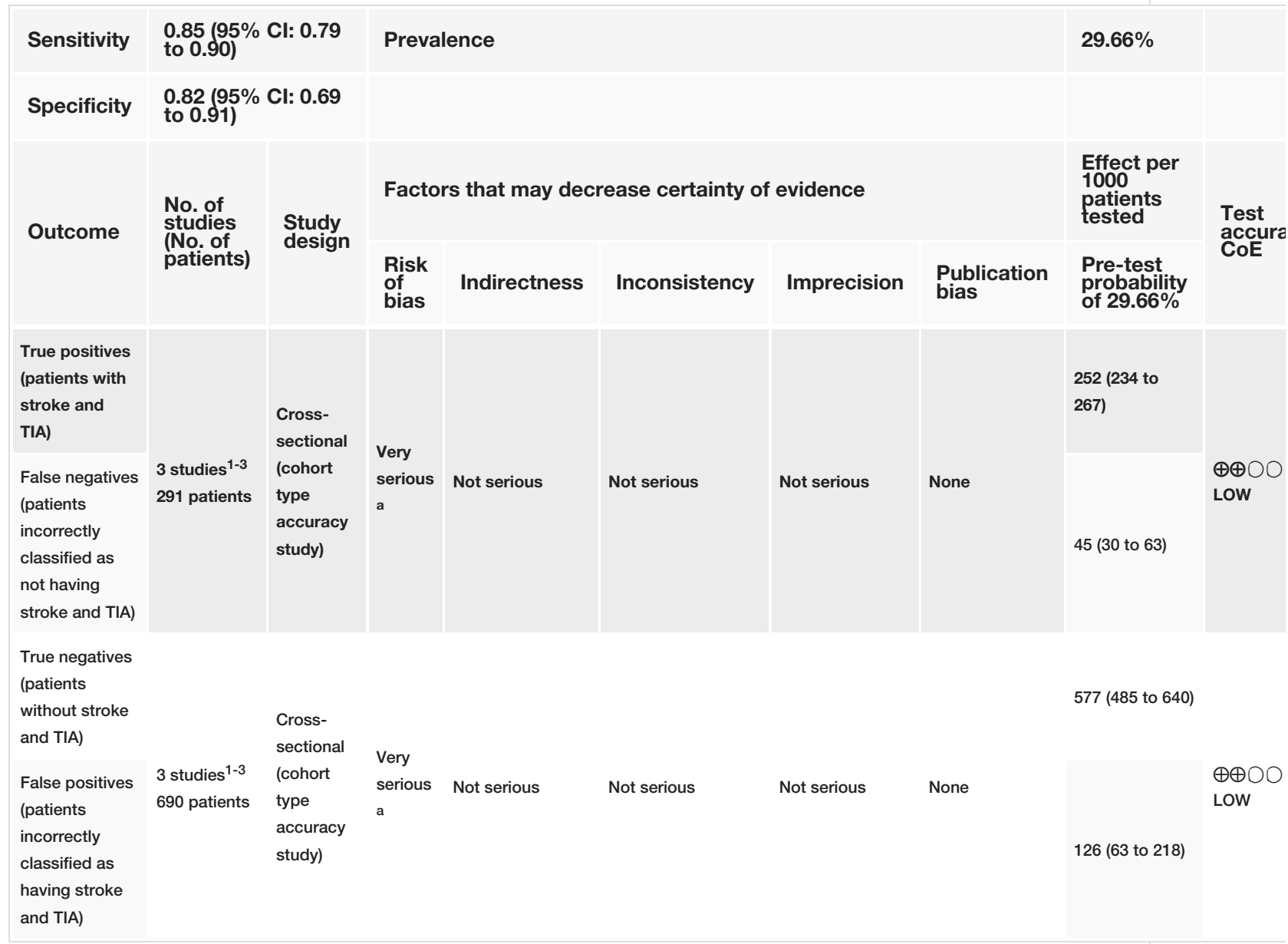

\section{TABLE 22: Evidence profile table for Melbourne Ambulance Stroke Scale (MASS)}

Question: Should MASS be used to diagnose stroke and TIA in patients suspected of stroke?

Bibliography: Bergs, 2010 [14]; Bray, 2005 [15]; Bray, 2010 [28]

Explanations: a- serious risk of bias due to patient selection and unclear risk of bias due to reference standard and flow and timing

TIA: Transient ischemic attack

\section{Additional Information}

Disclosures

Human subjects: All authors have confirmed that this study did not involve human participants or tissue. Animal subjects: All authors have confirmed that this study did not involve animal subjects or tissue. Conflicts of interest: In compliance with the ICMJE uniform disclosure form, all authors declare the following: Payment/services info: Funding for the submission fees to CUREUS.COM was provided by the French Red Cross and the Global First Aid Reference Center. No other funding was requested or required for this review. Financial relationships: All authors have declared that they have no financial relationships at present or within the previous three years with any organizations that might have an interest in the submitted work. Other relationships: All authors have declared that there are no other relationships or activities that could appear to have influenced the submitted work.

\section{Acknowledgements}

The authors would like to thank Vere Bora $\mathrm{PhD}$, researcher at the Centre for Evidence-Based Practice $(\mathrm{CEBaP})$, Belgian Red Cross for help with performing the literature searches, and Eddy Lang, MD for his methodological expertise. The authors would like to express their appreciation to the other members of the International Liaison Committee on Resuscitation First Aid Task Force who met authorship criteria as a collaborator: Vere Borra, Jestin N. Carlson, Nathan P. Charlton, David S. Markenson, Tetsuya Sakamoto, Janel M. Swain, Jeff A. Woodin, Matthew J Douma, Theresa Aves, Katherine Allan, Jason C. Bendall, David C. Berry, Wei-Tien Chang, Jonathan Epstein, Natalie Hood, Steve Lin. 


\section{References}

1. GBD 2015 Mortality and Causes of Death Collaborators: Global, regional, and national life expectancy, allcause mortality, and cause-specific mortality for 249 causes of death, 1980-2015: a systematic analysis for the Global Burden of Disease Study 2015. Lancet. 2016, 388:1459-1544. 10.1016/S0140-6736(16)31012-1

2. Singletary EM, Zideman DA, De Buck ED, et al.: Part 9: First aid: 2015 international consensus on first aid science with treatment recommendations. Circulation. 2015, 132:269-311. 10.1161/CIR.0000000000000278

3. Zideman DA, Singletary EM, De Buck ED, et al.: Part 9: First aid: 2015 international consensus on first aid science with treatment recommendations. Resuscitation. 2015, 95:225-261. 10.1016/j.resuscitation.2015.07.047

4. Higgins JP, Altman DG, Gotzsche PC, et al.: The Cochrane Collaboration's tool for assessing risk of bias in randomised trials. BMJ. 2011, 343:5928. 10.1136/bmj.d5928

5. Moher D, Liberati A, Tetzlaff J, Altman DG: Preferred reporting items for systematic reviews and metaanalyses: the PRISMA statement. BMJ. 2009, 339:2535. 10.1136/bmj.b2535

6. McHugh ML: Interrater reliability: the kappa statistic. Biochem Med (Zagreb). 2012, 22:276-282. 10.11613/BM.2012.031

7. Schunemann HJ, Cuello C, Akl EA, et al.: GRADE guidelines: 18. How ROBINS-I and other tools to assess risk of bias in nonrandomized studies should be used to rate the certainty of a body of evidence. J Clin Epidemiol. 2019, 111:105-114. 10.1016/j.jclinepi.2018.01.012

8. Whiting PF, Rutjes AW, Westwood ME, et al.: QUADAS-2: a revised tool for the quality assessment of diagnostic accuracy studies. Ann Intern Med. 2011, 155:529-536. 10.7326/0003-4819-155-8-20111018000009

9. GRADE Handbook. Schünemann H, Brożek J, Guyatt G, Oxman A (ed): The GRADE Working Group, 2013.

10. Schunemann HJ, Mustafa RA, Brozek J, et al.: GRADE guidelines: 21 part 2. Test accuracy: inconsistency, imprecision, publication bias, and other domains for rating the certainty of evidence and presenting it in evidence profiles and summary of findings tables. J Clin Epidemiol. 2020, 122:142-152. 10.1016/j.jclinepi.2019.12.021

11. Schunemann HJ, Mustafa RA, Brozek J, et al.: GRADE guidelines: 21 part 1. Study design, risk of bias, and indirectness in rating the certainty across a body of evidence for test accuracy. J Clin Epidemiol. 2020, 122:129-141. 10.1016/j.jclinepi.2019.12.020

12. Freeman SC, Kerby CR, Patel A, Cooper NJ, Quinn T, Sutton AJ: Development of an interactive web-based tool to conduct and interrogate meta-analysis of diagnostic test accuracy studies: MetaDTA. BMC Med Res Methodol. 2019, 19:81. 10.1186/s12874-019-0724-X

13. Andsberg G, Esbjornsson M, Olofsson A, Lindgren A, Norrving B, von Euler M: PreHospital ambulance stroke test - pilot study of a novel stroke test. Scand J Trauma Resusc Emerg Med. 2017, 25:37. 10.1186/s13049017-0377-X

14. Bergs J, Sabbe M, Moons P: Prehospital stroke scales in a Belgian prehospital setting: a pilot study . Eur J Emerg Med. 2010, 17:2-6. 10.1097/MEJ.0b013e32831040ec

15. Bray JE, Martin J, Cooper G, Barger B, Bernard S, Bladin C: Paramedic identification of stroke: community validation of the melbourne ambulance stroke screen. Cerebrovasc Dis. 2005, 20:28-33. 10.1159/000086201

16. Chen S, Sun H, Lei Y, et al.: Validation of the Los Angeles pre-hospital stroke screen (LAPSS) in a Chinese urban emergency medical service population. PLoS ONE. 2013, 8:70742. 10.1371/journal.pone.0070742

17. Fothergill RT, Williams J, Edwards MJ, Russell IT, Gompertz P: Does use of the recognition of stroke in the emergency room stroke assessment tool enhance stroke recognition by ambulance clinicians?. Stroke. 2013, 44:3007-3012. 10.1161/STROKEAHA.13.000851

18. Kidwell CS, Starkman S, Eckstein M, Weems K, Saver JL: Identifying stroke in the field. Prospective validation of the Los Angeles prehospital stroke screen (LAPSS). Stroke. 2000, 31:71-76. 10.1161/01.str.31.1.71

19. Kim DH, Kim SW, Jun SM, Kim JK: Accuracy of prehospital stroke recognition by paramedics and TPA therapy rate after transportation. J Neurol Sci. 2017, 381:867. 10.1016/j.jns.2017.08.2444

20. O'Brien W, Crimmins D, Donaldson W, Risti R, Clarke TA, Whyte S, Sturm J: FASTER (Face, Arm, Speech, Time, Emergency Response): experience of Central Coast Stroke Services implementation of a pre-hospital notification system for expedient management of acute stroke. J Clin Neurosci. 2012, 19:241-245. 10.1016/j.jocn.2011.06.009

21. Pickham D, Valdez A, Demeestere J, et al.: Prognostic value of BEFAST vs. FAST to identify stroke in a prehospital setting. Prehosp Emerg Care. 2019, 23:195-200. 10.1080/10903127.2018.1490837

22. Vanni S, Polidori G, Pepe G, Chiarlone M, Albani A, Pagnanelli A, Grifoni S: Use of biomarkers in triage of patients with suspected stroke. J Emerg Med. 2011, 40:499-505. 10.1016/j.jemermed.2008.09.028

23. Wall HK, Beagan BM, O'Neill J, Foell KM, Boddie-Willis CL: Addressing stroke signs and symptoms through public education: the Stroke Heroes Act FAST campaign. Prev Chronic Dis. 2008, 5:49.

24. Wojner-Alexandrov AW, Alexandrov AV, Rodriguez D, Persse D, Grotta JC: Houston paramedic and emergency stroke treatment and outcomes study (HoPSTO). Stroke. 2005, 36:1512-1518. 10.1161/01.STR.0000170700.45340.39

25. Kothari RU, Pancioli A, Liu T, Brott T, Broderick J: Cincinnati Prehospital Stroke Scale: reproducibility and validity. Ann Emerg Med. 1999, 33:373-378. 10.1016/s0196-0644(99)70299-4

26. Asimos AW, Ward S, Brice JH, Rosamond WD, Goldstein LB, Studnek J: Out-of-hospital stroke screen accuracy in a state with an emergency medical services protocol for routing patients to acute stroke centers. Ann Emerg Med. 2014, 64:509-515. 10.1016/j.annemergmed.2014.03.024

27. Berglund A, Svensson L, Wahlgren N, von Euler M: Face Arm Speech Time Test use in the prehospital setting, better in the ambulance than in the emergency medical communication center. Cerebrovasc Dis. 2014, 37:212-216. 10.1159/000358116

28. Bray JE, Coughlan K, Barger B, Bladin C: Paramedic diagnosis of stroke: examining long-term use of the Melbourne Ambulance Stroke Screen (MASS) in the field. Stroke. 2010, 41:1363-1366. 10.1161/STROKEAHA.109.571836

29. Chenkin J, Gladstone DJ, Verbeek PR, Lindsay P, Fang J, Black SE, Morrison L: Predictive value of the Ontario prehospital stroke screening tool for the identification of patients with acute stroke. Prehosp Emerg Care. 2009, 13:153-159. 10.1080/10903120802706146

30. English SW, Rabinstein AA, Mandrekar J, Klaas JP: Rethinking prehospital stroke notification: assessing utility of emergency medical services impression and Cincinnati Prehospital Stroke Scale. J Stroke 


\section{Cureus}

Cerebrovasc Dis. 2018, 27:919-925. 10.1016/j.jstrokecerebrovasdis.2017.10.036

31. Frendl DM, Strauss DG, Underhill BK, Goldstein LB: Lack of impact of paramedic training and use of the Cincinnati prehospital stroke scale on stroke patient identification and on-scene time. Stroke. 2009, 40:754756. 10.1161/STROKEAHA.108.531285

32. Greenberg K, Lesenskyj A, Eichorn D, Maxwell CR, D’Ambrosio M, Vezne-daroglu E, Binning MJ: Change takes time: EMS as the spark plug for faster acute ischemic stroke care. Mathews J Emerg Med. 2017, 2:025.

33. Harbison J, Hossain O, Jenkinson D, Davis J, Louw SJ, Ford GA: Diagnostic accuracy of stroke referrals from primary care, emergency room physicians, and ambulance staff using the face arm speech test. Stroke. 2003, 34:71-76. 10.1161/01.str.0000044170.46643.5e

34. Iguchi Y, Kimura K, Watanabe M, Shibazaki K, Aoki J: Utility of the Kurashiki Prehospital Stroke Scale for hyperacute stroke. Cerebrovasc Dis. 2011, 31:51-56. 10.1159/000320854

35. Ramanujam P, Guluma KZ, Castillo EM, et al.: Accuracy of stroke recognition by emergency medical dispatchers and paramedics--San Diego experience. Prehosp Emerg Care. 2008, 12:307-313. 10.1080/10903120802099526

36. Studnek JR, Asimos A, Dodds J, Swanson D: Assessing the validity of the Cincinnati Prehospital Stroke Scale and the medic prehospital assessment for code stroke in an urban emergency medical services agency. Prehosp Emerg Care. 2013, 17:348-353. 10.3109/10903127.2013.773113

37. Brandler ES, Sharma M, Sinert RH, Levine SR: Prehospital stroke scales in urban environments: a systematic review. Neurology. 2014, 82:2241-2249. 10.1212/WNL.0000000000000523

38. Rudd M, Buck D, Ford GA, Price CI: A systematic review of stroke recognition instruments in hospital and prehospital settings. Emerg Med J. 2016, 33:818-822. 10.1136/emermed-2015-205197

39. Zhelev Z, Walker G, Henschke N, Fridhandler J, Yip S: Prehospital stroke scales as screening tools for early identification of stroke and transient ischemic attack. Cochrane Database Syst Rev. 2019, 4:CD011427. 10.1002/14651858.CD011427.pub2

40. Liferidge AT, Brice JH, Overby BA, Evenson KR: Ability of laypersons to use the Cincinnati Prehospital Stroke Scale. Prehosp Emerg Care. 2004, 8:384-387. 10.1016/j.prehos.2004.05.004 